\title{
Global Stability of Humoral Immunity HIV Infection Models with Chronically Infected Cells and Discrete Delays
}

\author{
A. M. Elaiw and N. A. Alghamdi \\ Department of Mathematics, Faculty of Science, King Abdulaziz University, P.O. Box 80203, Jeddah 21589, Saudi Arabia \\ Correspondence should be addressed to A. M. Elaiw; a_m_elaiw@yahoo.com
}

Received 13 May 2015; Revised 7 August 2015; Accepted 25 August 2015

Academic Editor: Zizhen Zhang

Copyright (c) 2015 A. M. Elaiw and N. A. Alghamdi. This is an open access article distributed under the Creative Commons Attribution License, which permits unrestricted use, distribution, and reproduction in any medium, provided the original work is properly cited.

\begin{abstract}
We study the global stability of three HIV infection models with humoral immune response. We consider two types of infected cells: the first type is the short-lived infected cells and the second one is the long-lived chronically infected cells. In the three HIV infection models, we modeled the incidence rate by bilinear, saturation, and general forms. The models take into account two types of discrete-time delays to describe the time between the virus entering into an uninfected $\mathrm{CD} 4^{+} \mathrm{T}$ cell and the emission of new active viruses. The existence and stability of all equilibria are completely established by two bifurcation parameters, $R_{0}$ and $R_{1}$. The global asymptotic stability of the steady states has been proven using Lyapunov method. In case of the general incidence rate, we have presented a set of sufficient conditions which guarantee the global stability of model. We have presented an example and performed numerical simulations to confirm our theoretical results.
\end{abstract}

\section{Introduction}

During last decades, many researchers have developed and analyzed several mathematical models which describe human immunodeficiency virus (HIV) dynamics (see, e.g., [1-12]). HIV mainly targets the $\mathrm{CD} 4^{+} \mathrm{T}$ cells, leading to Acquired Immunodeficiency Syndrome (AIDS). Most of the HIV mathematical models presented in the literature consider only one type of infected cells called short-lived infected cells. However, it was shown that there is another source for the virus which is called long-lived chronically infected cells. This type of cells generates smaller number of viruses than the short-lived infected cells, but it lives longer [4]. The basic HIV dynamics model with long-lived chronically infected cells presented in [4] is given by

$$
\begin{aligned}
\dot{T} & =\pi-(1-\varepsilon) k T V-d T, \\
\dot{T}^{*} & =(1-\xi)(1-\varepsilon) k T V-b T^{*}, \\
\dot{C}^{*} & =\xi(1-\varepsilon) k T V-a C^{*}, \\
\dot{V} & =N_{T} b T^{*}+N_{C} a C^{*}-c V .
\end{aligned}
$$

Here $T, T^{*}, C^{*}$, and $V$ are the concentrations of the uninfected $\mathrm{CD} 4^{+} \mathrm{T}$ cells, short-lived infected $\mathrm{CD} 4^{+} \mathrm{T}$ cells, long-lived chronically infected $\mathrm{CD} 4^{+} \mathrm{T}$ cells, and free virus particles, respectively. $\pi$ represents birth rate constant of the uninfected $\mathrm{CD}^{+}{ }^{+} \mathrm{T}$ cells. $k$ is the infection rate constant. Parameters $d, b, a$, and $c$ are the death rate constants of uninfected $\mathrm{CD}^{+}{ }^{+} \mathrm{T}$ cells, short-lived infected $\mathrm{CD} 4^{+} \mathrm{T}$ cells, long-lived chronically infected $\mathrm{CD} 4^{+} \mathrm{T}$ cells, and free viruses, respectively. The fractions $\xi$ and $(1-\xi)$ with $0<\xi<1$ are the probabilities that, upon infection, an uninfected $\mathrm{CD} 4^{+} \mathrm{T}$ cell will become either long-lived chronically infected or shortlived infected. $N_{T}$ and $N_{C}$ denote the average numbers of free virus particles produced in the lifetime of the short-lived infected and long-lived chronically infected cells, respectively. Model (1) incorporates reverse transcriptase inhibitor drugs with drug efficacy $\varepsilon$ and $0 \leq \varepsilon<1$.

In model (1), the immune response has not been modeled. The immune response plays an important role in controlling the diseases. In reality, the immune response needs indispensable components to do its job such as antibodies, cytokines, natural killer cells, and $\mathrm{T}$ cells. The antibody immune response is a part of the adaptive system in which 
the body responds to pathogens by primarily using the antibodies which are generated by the B cells, while the other part is the Cytotoxic T Lymphocytes (CTL) immune response where the CTL attacks and kills the infected cells [3]. In malaria disease, the humoral immune response is more effective than the CTL immune response. In the virus dynamics literature, several models have considered the effect of CTL immune response $[3,13]$ or the humoral immune response [14-16]. Obaid and Elaiw [15] proposed the following model which takes into consideration the humoral immune response:

$$
\begin{aligned}
\dot{T} & =\pi-(1-\varepsilon) k T V-d T, \\
\dot{T}^{*} & =(1-\xi)(1-\varepsilon) k T V-b T^{*}, \\
\dot{C}^{*} & =\xi(1-\varepsilon) k T V-a C^{*}, \\
\dot{V} & =N_{T} b T^{*}+N_{C} a C^{*}-r V Z-c V, \\
\dot{Z} & =g V Z-\mu Z .
\end{aligned}
$$

Here, the variable $Z$ represents the concentration of B cells. The HIV are attacked by the B cells at rate $r V Z$. The terms $g V Z$ and $\mu Z$ represent the proliferation and death rates of the $B$ cells, respectively. In model (2), it is assumed that once the HIV contacts the CD $4^{+} \mathrm{T}$ cell, it becomes infected producing new viruses. Actually, there exists an intracellular time delay between the time the HIV contacts an uninfected $\mathrm{CD} 4^{+} \mathrm{T}$ cell and the time it becomes actively infected $\mathrm{CD} 4^{+} \mathrm{T}$ cell [17]. In the literature, several papers have proposed various HIV models with time delays [17-21].

Our aim in this work is to propose three HIV dynamics models with two types of infected cells, two types of intracellular delays, and humoral immunity. Bilinear and saturated incidences have been proposed in the first and second model, respectively, while a general nonlinear incidence rate is proposed in the third model. For each model, we derive two bifurcation parameters, $R_{0}$ and $R_{1}$, and establish the global stability using Lyapunov functional.

\section{Model with Bilinear Incidence}

We propose the following HIV infection model with humoral immunity, two types of infected cells and two types of intracellular delays:

$$
\begin{aligned}
\dot{T}(t)= & \pi-(1-\varepsilon) k T(t) V(t)-d T(t), \\
\dot{T}^{*}(t)= & e^{-m_{1} \tau_{1}}(1-\xi)(1-\varepsilon) k T\left(t-\tau_{1}\right) V\left(t-\tau_{1}\right) \\
& -b T^{*}(t), \\
\dot{C}^{*}(t)= & e^{-m_{1} \tau_{1}} \xi(1-\varepsilon) k T\left(t-\tau_{1}\right) V\left(t-\tau_{1}\right) \\
& -a C^{*}(t), \\
\dot{V}(t)= & e^{-m_{2} \tau_{2}} N_{T} b T^{*}\left(t-\tau_{2}\right)+e^{-m_{3} \tau_{3}} N_{C} a C^{*}\left(t-\tau_{3}\right) \\
& -r V(t) Z(t)-c V(t), \\
\dot{Z}(t)= & g V(t) Z(t)-\mu Z(t) .
\end{aligned}
$$

Parameter $\tau_{1}$ represents the time between HIV contact with an uninfected $\mathrm{CD} 4^{+} \mathrm{T}$ cell and the cell becoming infected but not yet producer cell. The factor $e^{-m_{1} \tau_{1}}$ represents the loss of $\mathrm{CD}^{+} \mathrm{T}$ cells during the interval $\left[t-\tau_{1}, t\right]$. The parameters $\tau_{2}$ and $\tau_{3}$ represent the time necessary for producing new infectious viruses from the short-lived infected and longlived chronically infected cells, respectively. The factors $e^{-m_{2} \tau_{2}}$ and $e^{-m_{3} \tau_{3}}$ represent the loss of short-lived and long-lived chronically infected cells during the intervals $\left[t-\tau_{2}, t\right]$ and $\left[t-\tau_{3}, t\right]$, respectively. Here, $m_{1}, m_{2}$, and $m_{3}$ are positive constants.

The initial conditions for system (2) are given by

$$
\begin{aligned}
T(u) & =\varphi_{1}(u), \\
T^{*}(u) & =\varphi_{2}(u), \\
C^{*}(u) & =\varphi_{3}(u), \\
V(u) & =\varphi_{4}(u), \\
Z(u) & =\varphi_{5}(u) \\
\varphi_{j}(u) & \geqslant 0, \quad u \in[-\tau, 0), j=1,2, \ldots, 5, \\
\varphi_{j}(0) & >0, \quad j=1,2, \ldots, 5,
\end{aligned}
$$

where $\left(\varphi_{1}(u), \varphi_{2}(u), \ldots, \varphi_{5}(u)\right) \in C\left([-\tau .0), R_{+}^{5}\right)$, where $C$ is the Banach space of continuous functions and $\tau=$ $\max \left\{\tau_{1}, \tau_{2}, \tau_{3}\right\}$. We note that system (3)-(7) with initial conditions (8) has a unique solution satisfying $t$ [22].

\subsection{Positive Invariance}

Proposition 1. The solution $\left(T(t), T^{*}(t), C^{*}(t), V(t), Z(t)\right)$ of (3)-(7) with initial conditions (8) is nonnegative for $t \geqslant 0$ and ultimately bounded.

Proof. Assume that $T(t) \leq 0$ on $[0, \rho]$ for some constant $\rho$ and $T\left(t^{*}\right)=0, t^{*} \in[0, \rho]$. From (3), we get $\dot{T}\left(t^{*}\right)=\pi>0$ and hence $T(t)>0$, for some $t \in\left(t^{*}, t^{*}+\varrho\right)$, and sufficiently small $\varrho>0$. This leads to a contradiction; therefore, $T(t)>0$, for all $t \geqslant 0$. From (4), (5), and (6), we have

$$
\begin{gathered}
T^{*}(t)=\varphi_{2}(0) e^{-b t}+e^{-m_{1} \tau_{1}}(1-\xi)(1-\varepsilon) \\
\cdot k \int_{0}^{t} e^{-b(t-\eta)} T\left(\eta-\tau_{1}\right) V\left(\eta-\tau_{1}\right) d \eta, \\
C^{*}(t)=\varphi_{3}(0) e^{-a t}+e^{-m_{1} \tau_{1}} \xi(1-\varepsilon) \\
\cdot k \int_{0}^{t} e^{-a(t-\eta)} T\left(\eta-\tau_{1}\right) V\left(\eta-\tau_{1}\right) d \eta, \\
V(t)=\varphi_{4}(0) e^{-\int_{0}^{t}(c+r Z(\varsigma)) d \varsigma} \\
+e^{-m_{2} \tau_{2}} N_{T} b \int_{0}^{t} e^{-\int_{\eta}^{t}(c+r Z(\varsigma)) d \varsigma} T^{*}\left(\eta-\tau_{2}\right) d \eta \\
+e^{-m_{3} \tau_{3}} N_{C} a \int_{0}^{t} e^{-\int_{\eta}^{t}(c+r Z(\varsigma)) d \varsigma} C^{*}\left(\eta-\tau_{3}\right) d \eta .
\end{gathered}
$$


This confirms that $T^{*}(t) \geqslant 0, C^{*}(t) \geqslant 0$, and $V(t) \geqslant 0$, for all $t \in[0, \tau]$. By a recursive argument, we get that $T^{*}(t) \geqslant 0$, $C^{*}(t) \geqslant 0$, and $V(t) \geqslant 0$, for all $t \geqslant 0$. Moreover, from (7), we obtain

$$
Z(t)=\varphi_{5}(0) e^{-\int_{0}^{t}(\mu-g V(\varsigma)) d \varsigma}
$$

Clearly, $Z(t) \geqslant 0, t \geqslant 0$. Now, we let $G_{1}(t)=e^{-m_{1} \tau_{1}} T\left(t-\tau_{1}\right)+$ $T^{*}(t)+C^{*}(t), G_{2}(t)=V(t)+(r / g) Z(t), s_{1}=\min \{d, a, b\}$, $s_{2}=\min \{c, \mu\}, L_{1}=\pi / s_{1}, L_{2}=\left(N_{T} b+N_{C} a\right) L_{1} / s_{2}$, and $L_{3}=g L_{2} / r$; then,

$$
\begin{aligned}
\dot{G}_{1}(t)= & e^{-m_{1} \tau_{1}}\left[\pi-d T\left(t-\tau_{1}\right)\right]-b T^{*}(t)-a C^{*}(t) \\
\leq & \pi e^{-m_{1} \tau_{1}} \\
& -s_{1}\left(e^{-m_{1} \tau_{1}} T\left(t-\tau_{1}\right)+T^{*}(t)+C^{*}(t)\right) \\
= & \pi e^{-m_{1} \tau_{1}}-s_{1} G_{1}(t)<\pi-s_{1} G_{1}(t) .
\end{aligned}
$$

Hence, $\lim \sup _{t \rightarrow \infty} G_{1}(t) \leq L_{1}$. Since $T(t)>0, T^{*}(t) \geq$ 0 , and $C^{*}(t) \geq 0$, then $\limsup _{t \rightarrow \infty} T^{*}(t) \leq L_{1}$ and $\lim \sup _{t \rightarrow \infty} C^{*}(t) \leq L_{1}$. Also,

$$
\begin{aligned}
\dot{G}_{2}(t)= & e^{-m_{2} \tau_{2}} N_{T} b T^{*}\left(t-\tau_{2}\right) \\
& +e^{-m_{3} \tau_{3}} N_{C} a C^{*}\left(t-\tau_{3}\right)-c V(t)-\frac{r \mu}{g} Z(t) \\
\leq & \left(e^{-m_{2} \tau_{2}} N_{T} b+e^{-m_{3} \tau_{3}} N_{C} a\right) L_{1} \\
& -s_{2}\left(V(t)+\frac{r}{g} Z(t)\right) \\
< & \left(N_{T} b+N_{C} a\right) L_{1}-s_{2} G_{2}(t) .
\end{aligned}
$$

Hence, $\lim \sup _{t \rightarrow \infty} G_{2}(t) \leq L_{2}$. Since $V(t)$ and $Z(t)$ are nonnegative, then $\lim \sup _{t \rightarrow \infty} V(t) \leq L_{2}$ and $\limsup _{t \rightarrow \infty} Z(t) \leq$ $L_{3}$. Therefore, $T(t), T^{*}(t), C^{*}(t), V(t)$, and $Z(t)$ are ultimately bounded.

2.2. Steady States. System (3)-(7) always admits an uninfected steady state $S_{0}=(\pi / d, 0,0,0,0)$. Let $T_{0}=\pi / d$. Now, we define the basic reproduction number for system (3)-(7) as

$$
\begin{aligned}
& R_{0} \\
& =\frac{(1-\varepsilon) k T_{0}\left[e^{-\left(m_{1} \tau_{1}+m_{2} \tau_{2}\right)}(1-\xi) N_{T}+e^{-\left(m_{1} \tau_{1}+m_{3} \tau_{3}\right)} \xi N_{C}\right]}{c} .
\end{aligned}
$$

The system has two other steady states, infected steady state without humoral immune response $S_{1}=\left(T_{1}, T_{1}^{*}, C_{1}^{*}, V_{1}, 0\right)$ and infected steady state with humoral immune response $S_{2}=$ $\left(T_{2}, T_{2}^{*}, C_{2}^{*}, V_{2}, Z_{2}\right)$ :

$$
\begin{aligned}
T_{1} & =\frac{T_{0}}{R_{0}}, \\
T_{1}^{*} & =e^{-m_{1} \tau_{1}} \frac{(1-\xi) \pi}{b} \frac{\left(R_{0}-1\right)}{R_{0}}, \\
C_{1}^{*} & =e^{-m_{1} \tau_{1}} \frac{\xi \pi}{a} \frac{\left(R_{0}-1\right)}{R_{0}}, \\
V_{1} & =\frac{d}{(1-\varepsilon) k}\left(R_{0}-1\right) \\
T_{2} & =\frac{\pi g}{g d+(1-\varepsilon) k \mu}, \\
T_{2}^{*} & =e^{-m_{1} \tau_{1}} \frac{(1-\xi)(1-\varepsilon) k \pi \mu}{b(d g+(1-\varepsilon) k \mu)} \\
C_{2}^{*} & =e^{-m_{1} \tau_{1}} \frac{\xi(1-\varepsilon) k \pi \mu}{a(d g+(1-\varepsilon) k \mu)} \\
V_{2} & =\frac{\mu}{g}, \\
Z_{2} & =\frac{c}{r}\left(\frac{d g R_{0}}{d g+(1-\varepsilon) k \mu}-1\right) .
\end{aligned}
$$

We note that $T_{1}, T_{1}^{*}, C_{1}^{*}$, and $V_{1}$ are positive when $R_{0}>1$ and where $T_{2}, T_{2}^{*}, C_{2}^{*}$, and $V_{2}>0$ and $Z_{2}>0$ when $d g R_{0} /(d g+$ $(1-\varepsilon) k \mu)>1$. Now, we define humoral immune response reproduction number $R_{1}$ as

$$
R_{1}=\frac{R_{0}}{1+(1-\varepsilon) k \mu / d g} .
$$

Clearly, $R_{0}>R_{1}$. From above, we can state the following lemma.

Lemma 2. For system (3)-(7), one has the following:

(i) If $R_{0} \leq 1$, then the system has only one positive steady state $S_{0}$.

(ii) If $R_{1} \leq 1<R_{0}$, then the system has two positive steady states $S_{0}$ and $S_{1}$.

(iii) If $R_{1}>1$, then the system has three positive steady states $S_{0}, S_{1}$, and $S_{2}$.

2.3. Global Stability Analysis. We establish the global stability of all the steady states of system (3)-(7) employing the method of Lyapunov function. Let us define

$$
H(\theta)=\theta-1-\ln \theta .
$$

Theorem 3. For system (3)-(7), if $R_{0} \leq 1$, then $S_{0}$ is GAS. 
Proof. Define

$$
\begin{aligned}
U_{0}= & T_{0} H\left(\frac{T}{T_{0}}\right)+\eta_{1} T^{*}+\eta_{2} C^{*}+\eta_{3} V+\eta_{4} Z \\
& +\eta_{5} \int_{0}^{\tau_{1}} T(t-\theta) V(t-\theta) d \theta \\
& +\eta_{6} \int_{0}^{\tau_{2}} T^{*}(t-\theta) d \theta+\eta_{7} \int_{0}^{\tau_{3}} C^{*}(t-\theta) d \theta,
\end{aligned}
$$

where $\eta_{i}, i=1, \ldots, 7$, are positive constants satisfying the following equations:

$$
\begin{aligned}
e^{-m_{1} \tau_{1}}(1-\varepsilon) k\left((1-\xi) \eta_{1}+\xi \eta_{2}\right) & =\eta_{5}, \\
\eta_{6}-b \eta_{1} & =0, \\
\eta_{7}-a \eta_{2} & =0, \\
\eta_{6}-\eta_{3} e^{-m_{2} \tau_{2}} N_{T} b & =0, \\
\eta_{7}-\eta_{3} e^{-m_{3} \tau_{3}} N_{C} a & =0, \\
g \eta_{4}-r \eta_{3} & =0, \\
\eta_{5} & =(1-\varepsilon) k .
\end{aligned}
$$

The solution of (18) is given by

$$
\begin{aligned}
& \eta_{1}=\frac{N_{T} e^{m_{1} \tau_{1}+m_{3} \tau_{3}}}{e^{m_{3} \tau_{3}}(1-\xi) N_{T}+e^{m_{2} \tau_{2}} \xi N_{C}}, \\
& \eta_{2}=\frac{N_{C} e^{m_{1} \tau_{1}+m_{2} \tau_{2}}}{e^{m_{3} \tau_{3}}(1-\xi) N_{T}+e^{m_{2} \tau_{2}} \xi N_{C}}, \\
& \eta_{3}=\frac{e^{m_{1} \tau_{1}+m_{2} \tau_{2}+m_{3} \tau_{3}}}{e^{m_{3} \tau_{3}}(1-\xi) N_{T}+e^{m_{2} \tau_{2}} \xi N_{C}}, \\
& \eta_{4}=\frac{r e^{m_{1} \tau_{1}+m_{2} \tau_{2}+m_{3} \tau_{3}}}{g\left[e^{m_{3} \tau_{3}}(1-\xi) N_{T}+e^{m_{2} \tau_{2}} \xi N_{C}\right]}, \\
& \eta_{5}=(1-\varepsilon) k, \\
& \eta_{6}=\frac{b N_{T} e^{m_{1} \tau_{1}+m_{3} \tau_{3}}}{e^{m_{3} \tau_{3}}(1-\xi) N_{T}+e^{m_{2} \tau_{2}} \xi N_{C}}, \\
& \eta_{7}=\frac{a N_{C} e^{m_{1} \tau_{1}+m_{2} \tau_{2}}}{e^{m_{3} \tau_{3}}(1-\xi) N_{T}+e^{m_{2} \tau_{2}} \xi N_{C}} .
\end{aligned}
$$

The values of $\eta_{i}, i=1, \ldots, 7$, will be used through the paper. Calculating the derivative of $U_{0}$ along the solutions of system (3)-(7) and applying $\pi=T_{0} d$, we obtain

$$
\begin{aligned}
& \frac{d U_{0}}{d t}=\left(1-\frac{T_{0}}{T}\right)(\pi-(1-\varepsilon) k T V-d T) \\
& \quad+\eta_{1}\left(e^{-m_{1} \tau_{1}}(1-\xi)(1-\varepsilon) k T\left(t-\tau_{1}\right) V\left(t-\tau_{1}\right)\right.
\end{aligned}
$$

$$
\begin{aligned}
& \left.-b T^{*}\right)+\eta_{2}\left(e^{-m_{1} \tau_{1}} \xi(1-\varepsilon) k T\left(t-\tau_{1}\right) V\left(t-\tau_{1}\right)\right. \\
& \left.-a C^{*}\right)+\eta_{3}\left(e^{-m_{2} \tau_{2}} N_{T} b T^{*}\left(t-\tau_{2}\right)\right. \\
& \left.+e^{-m_{3} \tau_{3}} N_{C} a C^{*}\left(t-\tau_{3}\right)-r V Z-c V\right)+\eta_{4}(g V Z \\
& -\mu Z)+\eta_{5} T V-\eta_{5} T\left(t-\tau_{1}\right) V\left(t-\tau_{1}\right)+\eta_{6} T^{*} \\
& -\eta_{6} T^{*}\left(t-\tau_{2}\right)+\eta_{7} C^{*}-\eta_{7} C^{*}\left(t-\tau_{3}\right) .
\end{aligned}
$$

Then,

$$
\begin{aligned}
\frac{d U_{0}}{d t}= & \left(1-\frac{T_{0}}{T}\right)(\pi-d T)+(1-\varepsilon) k T_{0} V-\eta_{3} c V \\
& -\eta_{4} \mu Z \\
= & -d \frac{\left(T-T_{0}\right)^{2}}{T}+c \eta_{3}\left(R_{0}-1\right) V-\eta_{4} \mu Z .
\end{aligned}
$$

Therefore, if $R_{0} \leq 1$, then $d U_{0} / d t \leq 0$, for all $T, V, Z>0$. The solutions of system (3)-(7) limit $M$, the largest invariant subset of $\left\{d U_{0} / d t=0\right\}$ [22]. We note that $d U_{0} / d t=0$ if and only if $T(t)=T_{0}, V(t)=0$, and $Z(t)=0$. For each element of $M$, we have $V(t)=0$ and $Z(t)=0$; then, $\dot{V}(t)=0$ and

$$
\begin{aligned}
0 & =\dot{V}(t) \\
& =e^{-m_{2} \tau_{2}} N_{T} b T^{*}\left(t-\tau_{2}\right)+e^{-m_{3} \tau_{3}} N_{C} a C^{*}\left(t-\tau_{3}\right) .
\end{aligned}
$$

Since $T^{*}(t), C^{*}(t) \geq 0$, then $T^{*}(t)=C^{*}(t)=0$. Hence, $d U_{0} / d t=0$ if and only if $T(t)=T_{0}, T^{*}(t)=0, C^{*}(t)=0$, $V(t)=0$, and $Z(t)=0$. It follows from LaSalle's invariance principle (LIP) that $S_{0}$ is GAS when $R_{0} \leq 1$.

Theorem 4. For system (3)-(7), assume that $R_{1} \leq 1<R_{0}$; then, $S_{1}$ is GAS.

Proof. Define

$$
\begin{aligned}
U_{1}= & T_{1} H\left(\frac{T}{T_{1}}\right)+\eta_{1} T_{1}^{*} H\left(\frac{T^{*}}{T_{1}^{*}}\right)+\eta_{2} C_{1}^{*} H\left(\frac{C^{*}}{C_{1}^{*}}\right) \\
& +\eta_{3} V_{1} H\left(\frac{V}{V_{1}}\right)+\eta_{4} Z \\
& +\eta_{5} T_{1} V_{1} \int_{0}^{\tau_{1}} H\left(\frac{T(t-\theta) V(t-\theta)}{T_{1} V_{1}}\right) d \theta \\
& +\eta_{6} T_{1}^{*} \int_{0}^{\tau_{2}} H\left(\frac{T^{*}(t-\theta)}{T_{1}^{*}}\right) d \theta \\
& +\eta_{7} C_{1}^{*} \int_{0}^{\tau_{3}} H\left(\frac{C^{*}(t-\theta)}{C_{1}^{*}}\right) d \theta .
\end{aligned}
$$


Then, $d U_{1} / d t$ is given by

$$
\begin{aligned}
& \frac{d U_{1}}{d t}=\left(1-\frac{T_{1}}{T}\right)(\pi-(1-\varepsilon) k T V-d T)+\eta_{1}(1 \\
& \left.-\frac{T_{1}^{*}}{T^{*}}\right)\left(e^{-m_{1} \tau_{1}}(1-\xi)(1-\varepsilon) k T\left(t-\tau_{1}\right) V\left(t-\tau_{1}\right)\right. \\
& \left.-b T^{*}\right)+\eta_{2}\left(1-\frac{C_{1}^{*}}{C^{*}}\right) \\
& +\left(e^{-m_{1} \tau_{1}} \xi(1-\varepsilon) k T\left(t-\tau_{1}\right) V\left(t-\tau_{1}\right)\right. \\
& \left.-a C^{*}\right)+\eta_{3}\left(1-\frac{V_{1}}{V}\right)\left(e^{-m_{2} \tau_{2}} N_{T} b T^{*}\left(t-\tau_{2}\right)\right. \\
& \left.+e^{-m_{3} \tau_{3}} N_{C} a C^{*}\left(t-\tau_{3}\right)-r V Z-c V\right)+\eta_{4}(g V Z \\
& -\mu Z)+\eta_{5}\left[T V-T\left(t-\tau_{1}\right) V\left(t-\tau_{1}\right)\right. \\
& \left.+T_{1} V_{1} \ln \left(\frac{T\left(t-\tau_{1}\right) V\left(t-\tau_{1}\right)}{T V}\right)\right]+\eta_{6}\left[T^{*}\right. \\
& \left.-C^{*}\left(t-\tau_{2}\right)+T_{1}^{*} \ln \left(\frac{T^{*}\left(t-\tau_{2}\right)}{T^{*}}\right)\right]+\eta_{7}\left[C^{*}\right. \\
& \left.T_{1}^{*} \ln \left(\frac{C^{*}\left(t-\tau_{3}\right)}{C^{*}}\right)\right] \cdot
\end{aligned}
$$

Equation (24) can be simplified as

$$
\begin{aligned}
& \frac{d U_{1}}{d t} \quad \\
& =\left(1-\frac{T_{1}}{T}\right)(\pi-d T) \\
& \quad-e^{m_{1} \tau_{1}} \eta_{1}(1-\xi)(1-\varepsilon) k T\left(t-\tau_{1}\right) V\left(t-\tau_{1}\right) \frac{T_{1}^{*}}{T^{*}} \\
& +\eta_{1} b T_{1}^{*} \\
& \quad-e^{-m_{1} \tau_{1}} \eta_{2} \xi(1-\varepsilon) k T\left(t-\tau_{1}\right) V\left(t-\tau_{1}\right) \frac{C_{1}^{*}}{C^{*}} \\
& +\eta_{2} a C_{1}^{*}-b \eta_{1} \frac{T^{*}\left(t-\tau_{2}\right) V_{1}}{V} \\
& \left.\quad-a \eta_{2} \frac{C^{*}\left(t-\tau_{3}\right) V_{1}+c \eta_{3} V_{1}+r \eta_{3} V_{1} Z-\mu \eta_{4} Z}{V}\right) \\
& +\eta_{5} T_{1} V_{1} \ln \left(\frac{T\left(t-\tau_{1}\right) V\left(t-\tau_{1}\right)}{T V}\right) \\
& +\eta_{2} a C_{1}^{*} \ln \left(\frac{C^{*}\left(t-\tau_{3}\right)}{C^{*}}\right) \\
&
\end{aligned}
$$

$$
\text { Applying } \pi=d T_{1}+(1-\varepsilon) k T_{1} V_{1} \text {, we get }
$$

$$
\begin{aligned}
& \frac{d U_{1}}{d t}=\left(1-\frac{T_{1}}{T}\right)\left(d T_{1}-d T\right)+(1-\varepsilon) \\
& \cdot k T_{1} V_{1}\left(1-\frac{T_{1}}{T}\right)-e^{-m_{1} \tau_{1}} \eta_{1}(1-\xi)(1-\varepsilon) \\
& k T\left(t-\tau_{1}\right) V\left(t-\tau_{1}\right) \frac{T_{1}^{*}}{T^{*}}+\eta_{1} b T_{1}^{*} \\
& -e^{-m_{1} \tau_{1}} \eta_{2} \xi(1-\varepsilon) k T\left(t-\tau_{1}\right) V\left(t-\tau_{1}\right) \frac{C_{1}^{*}}{C^{*}} \\
& +\eta_{2} a C_{1}^{*}-b \eta_{1} \frac{T^{*}\left(t-\tau_{2}\right) V_{1}}{V}-a \eta_{2} \\
& \cdot \frac{C^{*}\left(t-\tau_{3}\right) V_{1}}{V}+c \eta_{3} V_{1}+r \eta_{3} V_{1} Z-\mu \eta_{4} Z \\
& +T_{1} V_{1} e^{-m_{1} \tau_{1}} \eta_{1}(1-\xi)(1-\varepsilon) k \\
& \cdot \ln \left(\frac{T\left(t-\tau_{1}\right) V\left(t-\tau_{1}\right)}{T V}\right) \\
& +T_{1} V_{1} e^{-m_{1} \tau_{1}} \eta_{2} \xi(1-\varepsilon) k \\
& \cdot \ln \left(\frac{T\left(t-\tau_{1}\right) V\left(t-\tau_{1}\right)}{T V}\right)+\eta_{1} b T_{1}^{*} \\
& \cdot \ln \left(\frac{T^{*}\left(t-\tau_{2}\right)}{T^{*}}\right)+\eta_{2} a C_{1}^{*} \\
& \cdot \ln \left(\frac{C^{*}\left(t-\tau_{3}\right)}{C^{*}}\right) \text {. }
\end{aligned}
$$

Using the steady state conditions for $S_{1}$,

$$
\begin{aligned}
(1-\xi)(1-\varepsilon) k T_{1} V_{1} & =e^{m_{1} \tau_{1}} b T_{1}^{*}, \\
\xi(1-\varepsilon) k T_{1} V_{1} & =e^{m_{1} \tau_{1}} a C_{1}^{*}, \\
c V_{1} & =e^{-m_{2} \tau_{2}} N_{T} b T_{1}^{*}+e^{-m_{3} \tau_{3}} N_{C} a C_{1}^{*},
\end{aligned}
$$

we get $(1-\varepsilon) k T_{1} V_{1}=\eta_{1} b T_{1}^{*}+\eta_{2} a C_{1}^{*}$ and

$$
\begin{aligned}
\frac{d U_{1}}{d t}= & -d \frac{\left(T-T_{1}\right)^{2}}{T}+\eta_{1} b T_{1}^{*}\left(1-\frac{T_{1}}{T}\right) \\
& +\eta_{2} a C_{1}^{*}\left(1-\frac{T_{1}}{T}\right) \\
& -\eta_{1} b T_{1}^{*} \frac{T\left(t-\tau_{1}\right) V\left(t-\tau_{1}\right) T_{1}^{*}}{T_{1} V_{1} T^{*}}+\eta_{1} b T_{1}^{*} \\
& -\eta_{2} a C_{1}^{*} \frac{T\left(t-\tau_{1}\right) V\left(t-\tau_{1}\right) C_{1}^{*}}{T_{1} V_{1} C^{*}}+\eta_{2} a C_{1}^{*} \\
& -\eta_{1} b T_{1}^{*} \frac{V_{1} T^{*}\left(t-\tau_{2}\right)}{V T_{1}^{*}}
\end{aligned}
$$




$$
\begin{aligned}
& -\eta_{2} a C_{1}^{*} \frac{C^{*}\left(t-\tau_{3}\right) V_{1}}{V C_{1}^{*}}+\eta_{1} b T_{1}^{*}+\eta_{2} a C_{1}^{*} \\
& +r \eta_{3} V_{1} Z-\mu \eta_{4} Z \\
& +\eta_{1} b T_{1}^{*} \ln \left(\frac{T\left(t-\tau_{1}\right) V\left(t-\tau_{1}\right)}{T V}\right) \\
& +\eta_{2} a C_{1}^{*} \ln \left(\frac{T\left(t-\tau_{1}\right) V\left(t-\tau_{1}\right)}{T V}\right) \\
& +\eta_{1} b T_{1}^{*} \ln \left(\frac{T^{*}\left(t-\tau_{2}\right)}{T^{*}}\right) \\
& +\eta_{2} a C_{1}^{*} \ln \left(\frac{C^{*}\left(t-\tau_{3}\right)}{C^{*}}\right)
\end{aligned}
$$

Consider the following equalities:

$$
\begin{aligned}
& \ln (\left.\frac{T\left(t-\tau_{1}\right) V\left(t-\tau_{1}\right)}{T V}\right) \\
&= \ln \left(\frac{T_{j}}{T}\right)+\ln \left(\frac{T\left(t-\tau_{1}\right) V\left(t-\tau_{1}\right) T_{j}^{*}}{T_{j} V_{j} T^{*}}\right) \\
&+\ln \left(\frac{V_{j} T^{*}}{V T_{j}^{*}}\right), \\
& \ln \left(\frac{T\left(t-\tau_{1}\right) V\left(t-\tau_{1}\right)}{T V}\right) \\
&=\ln \left(\frac{T_{j}}{T}\right)+\ln \left(\frac{T\left(t-\tau_{1}\right) V\left(t-\tau_{1}\right) C_{j}^{*}}{T_{j} V_{j} C^{*}}\right) \\
&=\ln \left(\frac{C^{*}\left(t-\tau_{3}\right) V_{j}}{V C_{j}^{*}}\right)+\ln \left(\frac{V C_{j}^{*}}{V_{j} C^{*}}\right), \\
& \ln \left(\frac{C^{*}\left(t-\tau_{3}\right)}{C^{*}}\right) \\
&=\ln \left(\frac{T^{*}\left(t-\tau_{2}\right) V_{j}}{V C_{j}^{*}}\right)+\ln \left(\frac{V T_{j}^{*}}{V_{j} T^{*}}\right), \\
&\ln ) \\
&\left.\quad \frac{T^{*}}{C^{*}}\right) \\
&=
\end{aligned}
$$

$$
j=1,2 \text {. }
$$

Using (29) in case of $j=1$, we obtain

$$
\begin{gathered}
\frac{d U_{1}}{d t}=-d \frac{\left(T-T_{1}\right)^{2}}{T}-\eta_{1} b T_{1}^{*}\left[\frac{T_{1}}{T}-1-\ln \left(\frac{T_{1}}{T}\right)\right] \\
-\eta_{2} a C_{1}^{*}\left[\frac{T_{1}}{T}-1-\ln \left(\frac{T_{1}}{T}\right)\right]
\end{gathered}
$$

$$
\begin{aligned}
& -\eta_{1} b T_{1}^{*}\left[\frac{T\left(t-\tau_{1}\right) V\left(t-\tau_{1}\right) T_{1}^{*}}{T_{1} V_{1} T^{*}}-1\right. \\
& \left.-\ln \left(\frac{T\left(t-\tau_{1}\right) V\left(t-\tau_{1}\right) T_{1}^{*}}{T_{1} V_{1} T^{*}}\right)\right] \\
& -\eta_{2} a C_{1}^{*}\left[\frac{T\left(t-\tau_{1}\right) V\left(t-\tau_{1}\right) C_{1}^{*}}{T_{1} V_{1} C^{*}}-1\right. \\
& \left.-\ln \left(\frac{T\left(\mathrm{t}-\tau_{1}\right) V\left(t-\tau_{1}\right) C_{1}^{*}}{T_{1} V_{1} C^{*}}\right)\right] \\
& -\eta_{1} b T_{1}^{*}\left[\frac{T^{*}\left(t-\tau_{2}\right) V_{1}}{V T_{1}^{*}}-1\right. \\
& \left.-\ln \left(\frac{T^{*}\left(t-\tau_{2}\right) V_{1}}{V T_{1}^{*}}\right)\right]-\eta_{2} a C_{1}^{*}\left[\frac{C^{*}\left(t-\tau_{3}\right) V_{1}}{V C_{1}^{*}}\right. \\
& \left.-1-\ln \left(\frac{C^{*}\left(t-\tau_{3}\right) V_{1}}{V C_{1}^{*}}\right)\right]+r \eta_{3}\left(V_{1}-\frac{\mu}{g}\right) Z \\
& =-d \frac{\left(T-T_{1}\right)^{2}}{T}-\eta_{1} b T_{1}^{*}\left[H\left(\frac{T_{1}}{T}\right)\right. \\
& +H\left(\frac{T\left(t-\tau_{1}\right) V\left(t-\tau_{1}\right) T_{1}^{*}}{T_{1} V_{1} T^{*}}\right) \\
& \left.+H\left(\frac{T^{*}\left(t-\tau_{2}\right) V_{1}}{V T_{1}^{*}}\right)\right]-\eta_{2} a C_{1}^{*}\left[H\left(\frac{T_{1}}{T}\right)\right. \\
& +H\left(\frac{T\left(t-\tau_{1}\right) V\left(t-\tau_{1}\right) C_{1}^{*}}{T_{1} V_{1} C^{*}}\right) \\
& \left.+H\left(\frac{C^{*}\left(t-\tau_{3}\right) V_{1}}{V C_{1}^{*}}\right)\right]+r \eta_{3}\left(\frac{d g+\mu(1-\varepsilon) k}{g(1-\varepsilon) k}\right) \\
& \text { - }\left[R_{1}-1\right] Z \text {. }
\end{aligned}
$$

We have if $R_{0}>1$, then $T_{1}, T_{1}^{*}, C_{1}^{*}, V_{1}>0$. Since $R_{1} \leq 1$, then for all $T, T^{*}, C^{*}, V, Z>0$, we have $d U_{1} / d t \leq 0$. We note that $d U_{1} / d t=0$ at $S_{1}$. Then, from LIP, $S_{1}$ is GAS.

Theorem 5. For system (3)-(7), assume that $R_{1}>1$; then, $S_{2}$ is GAS.

Proof. We consider

$$
\begin{aligned}
U_{2}= & T_{2} H\left(\frac{T}{T_{2}}\right)+\eta_{1} T_{2}^{*} H\left(\frac{\mathrm{T}^{*}}{T_{2}^{*}}\right)+\eta_{2} C_{2}^{*} H\left(\frac{C^{*}}{C_{2}^{*}}\right) \\
& +\eta_{3} V_{2} H\left(\frac{V}{V_{2}}\right)+\eta_{4} Z_{2} H\left(\frac{Z}{Z_{2}}\right) \\
& +\eta_{5} T_{2} V_{2} \int_{0}^{\tau_{1}} H\left(\frac{T(t-\theta) V(t-\theta)}{T_{2} V_{2}}\right) d \theta
\end{aligned}
$$




$$
\begin{aligned}
& +\eta_{6} T_{2}^{*} \int_{0}^{\tau_{2}} H\left(\frac{T^{*}(t-\theta)}{T_{2}^{*}}\right) d \theta \\
& +\eta_{7} C_{2}^{*} \int_{0}^{\tau_{3}} H\left(\frac{C^{*}(t-\theta)}{C_{2}^{*}}\right) d \theta .
\end{aligned}
$$

Function $U_{2}$ along the trajectories of system (3)-(7) satisfies

$$
\begin{aligned}
& \frac{d U_{2}}{d t}=\left(1-\frac{T_{2}}{T}\right)(\pi-(1-\varepsilon) k T V-d T)+\eta_{1}(1 \\
& \left.-\frac{T_{2}^{*}}{T^{*}}\right)\left(e^{-m_{1} \tau_{1}}(1-\xi)(1-\varepsilon) k T\left(t-\tau_{1}\right) V\left(t-\tau_{1}\right)\right. \\
& \left.-b T^{*}\right)+\eta_{2}\left(1-\frac{C_{2}^{*}}{C^{*}}\right) \\
& \cdot\left(e^{-m_{1} \tau_{1}} \xi(1-\varepsilon) k T\left(t-\tau_{1}\right) V\left(t-\tau_{1}\right)\right. \\
& \left.-a C^{*}\right)+\eta_{3}\left(1-\frac{V_{2}}{V}\right)\left(e^{-m_{2} \tau_{2}} N_{T} b T^{*}\left(t-\tau_{2}\right)\right. \\
& \left.+e^{-m_{3} \tau_{3}} N_{C} a C^{*}\left(t-\tau_{3}\right)-r V Z-c V\right)+\eta_{4}(1 \\
& \left.-\frac{Z_{2}}{Z}\right)(g V Z-\mu Z)+\eta_{5}[T V \\
& -T\left(t-\tau_{1}\right) V\left(t-\tau_{1}\right) \\
& \left.+T_{2} V_{2} \ln \left(\frac{T\left(t-\tau_{1}\right) V\left(t-\tau_{1}\right)}{T V}\right)\right]+\eta_{6}\left[T^{*}\right. \\
& \left.-T^{*}\left(t-\tau_{2}\right)+T_{2}^{*} \ln \left(\frac{T^{*}\left(t-\tau_{2}\right)}{T^{*}}\right)\right]+\eta_{7}\left[C^{*}\right. \\
& \left.-C^{*}\left(t-\tau_{3}\right)+C_{2}^{*} \ln \left(\frac{C^{*}\left(t-\tau_{3}\right)}{C^{*}}\right)\right] .
\end{aligned}
$$

Using the steady state conditions for $S_{2}$,

$$
\begin{aligned}
\pi & =d T_{2}+(1-\varepsilon) k T_{2} V_{2}, \\
(1-\xi)(1-\varepsilon) k T_{2} V_{2} & =e^{m_{1} \tau_{1}} b T_{2}^{*}, \\
\xi(1-\varepsilon) k T_{2} V_{2}= & e^{m_{1} \tau_{1}} a C_{2}^{*}, \\
c V_{2}= & e^{-m_{2} \tau_{2}} N_{T} b T_{2}^{*}+e^{-m_{3} \tau_{3}} N_{C} a C_{2}^{*} \\
& -r V_{2} Z_{2},
\end{aligned}
$$

we get $(1-\varepsilon) k T_{2} V_{2}=\eta_{1} b T_{2}^{*}+\eta_{2} a C_{2}^{*}$ and

$$
\begin{aligned}
\frac{d U_{2}}{d t}= & -d \frac{\left(T-T_{2}\right)^{2}}{T}+\eta_{1} b T_{2}^{*}\left(1-\frac{T_{2}}{T}\right) \\
& +\eta_{2} a C_{2}^{*}\left(1-\frac{T_{2}}{T}\right) \\
& -\eta_{1} b T_{2}^{*} \frac{T\left(t-\tau_{1}\right) V\left(t-\tau_{1}\right) T_{2}^{*}}{T_{2} V_{2} T^{*}}+\eta_{1} b T_{2}^{*}
\end{aligned}
$$

$$
\begin{aligned}
& -\eta_{2} a C_{2}^{*} \frac{T\left(t-\tau_{1}\right) V\left(t-\tau_{1}\right) C_{2}^{*}}{T_{2} V_{2} C^{*}}+\eta_{2} a C_{2}^{*} \\
& -\eta_{1} b T_{2}^{*} \frac{V_{2} T^{*}\left(t-\tau_{2}\right)}{V T_{2}^{*}}-\eta_{2} a C_{2}^{*} \frac{V_{2} C^{*}\left(t-\tau_{3}\right)}{V C_{2}^{*}} \\
& +\eta_{1} b T_{2}^{*}+\eta_{2} a C_{2}^{*} \\
& +\eta_{1} b T_{2}^{*} \ln \left(\frac{T\left(t-\tau_{1}\right) V\left(t-\tau_{1}\right)}{T V}\right) \\
& +\eta_{2} a C_{2}^{*} \ln \left(\frac{T\left(t-\tau_{1}\right) V\left(t-\tau_{1}\right)}{T V}\right) \\
& +\eta_{1} b T_{2}^{*} \ln \left(\frac{T^{*}\left(t-\tau_{2}\right)}{T^{*}}\right) \\
& +\eta_{2} a C_{2}^{*} \ln \left(\frac{C^{*}\left(t-\tau_{3}\right)}{C^{*}}\right) .
\end{aligned}
$$

Using (29) in case of $j=2$, we obtain

$$
\begin{aligned}
& \frac{d U_{2}}{d t}=-d \frac{\left(T-T_{2}\right)^{2}}{T}-\eta_{1} b T_{2}^{*}\left[H\left(\frac{T_{2}}{T}\right)\right. \\
& +H\left(\frac{T\left(t-\tau_{1}\right) V\left(t-\tau_{1}\right) T_{2}^{*}}{T_{2} V_{2} T^{*}}\right) \\
& \left.+H\left(\frac{T^{*}\left(t-\tau_{2}\right) V_{2}}{V T_{2}^{*}}\right)\right]-\eta_{2} a C_{2}^{*}\left[H\left(\frac{T_{2}}{T}\right)\right. \\
& +H\left(\frac{T\left(t-\tau_{1}\right) V\left(t-\tau_{1}\right) C_{2}^{*}}{T_{2} V_{2} C^{*}}\right) \\
& \left.+H\left(\frac{C^{*}\left(t-\tau_{3}\right) V_{2}}{V C_{2}^{*}}\right)\right] .
\end{aligned}
$$

Since $R_{1}>1$, then $T_{2}, T_{2}^{*}, C_{2}^{*}, V_{2}$, and $Z_{2}>0$. It is observed that $d U_{2} / d t=0$ if and only if $T=T_{2}, T^{*}=T_{2}^{*}, C^{*}=C_{2}^{*}$, and $V=V_{2}$. Therefore, if $V=V_{2}$, then $\dot{V}=0$ and (6) becomes

$$
0=e^{-m_{2} \tau_{2}} N_{T} b T_{2}^{*}+e^{-m_{3} \tau_{3}} N_{C} a C_{2}^{*}-c V_{2}-r V_{2} Z,
$$

which gives $Z=Z_{2}$. Hence, $d U_{2} / d t$ is equal to zero at $S_{2}$. The global stability of $S_{2}$ follows from LIP.

\section{HIV Dynamics Model with Saturated Incidence}

We present an HIV infection model with saturated incidence:

$$
\begin{aligned}
\dot{T}(t) & =\pi-\frac{(1-\varepsilon) k T(t) V(t)}{1+\beta V(t)}-d T(t), \\
\dot{T}^{*}(t) & =e^{-m_{1} \tau_{1}} \frac{(1-\xi)(1-\varepsilon) k T\left(t-\tau_{1}\right) V\left(t-\tau_{1}\right)}{1+\beta V\left(t-\tau_{1}\right)}
\end{aligned}
$$




$$
\begin{aligned}
& -b T^{*}(t), \\
\dot{C}^{*}(t)= & e^{-m_{1} \tau_{1}} \frac{\xi(1-\varepsilon) k T\left(t-\tau_{1}\right) V\left(t-\tau_{1}\right)}{1+\beta V\left(t-\tau_{1}\right)} \\
& -a C^{*}(t), \\
\dot{V}(t)= & e^{-m_{2} \tau_{2}} N_{T} b T^{*}\left(t-\tau_{2}\right) \\
& +e^{-m_{3} \tau_{3}} N_{C} a C^{*}\left(t-\tau_{3}\right)-r V(t) Z(t) \\
& -c V(t), \\
\dot{Z}(t)= & g V(t) Z(t)-\mu Z(t),
\end{aligned}
$$

where $\beta>0$ is the saturation incidence rate constant. Similar to the previous section, one can show that the solutions of the model are nonnegative and bounded.

3.1. Steady States. System (37) always admits an uninfected steady state $S_{0}=\left(T_{0}, 0,0,0,0\right)$, where $T_{0}=\pi / d$. Now, we define the basic reproduction number for system (37) as

$$
\begin{aligned}
& R_{0} \\
& =\frac{(1-\varepsilon) k T_{0}\left[e^{-\left(m_{1} \tau_{1}+m_{2} \tau_{2}\right)}(1-\xi) N_{T}+e^{-\left(m_{1} \tau_{1}+m_{3} \tau_{3}\right)} \xi N_{C}\right]}{c} .
\end{aligned}
$$

The system has two other steady states $S_{1}\left(T_{1}, T_{1}^{*}, C_{1}^{*}, V_{1}, 0\right)$ and $S_{2}\left(T_{2}, T_{2}^{*}, C_{2}^{*}, V_{2}, Z_{2}\right)$, where

$$
\begin{aligned}
& T_{1}=\frac{\beta \pi\left[e^{-\left(m_{1} \tau_{1}+m_{2} \tau_{2}\right)}(1-\xi) N_{T}+e^{-\left(m_{1} \tau_{1}+m_{3} \tau_{3}\right)} \xi N_{C}\right]+c}{((1-\varepsilon) k+d \beta)\left[e^{-\left(m_{1} \tau_{1}+m_{2} \tau_{2}\right)}(1-\xi) N_{T}+e^{-\left(m_{1} \tau_{1}+m_{3} \tau_{3}\right)} \xi N_{C}\right]}, \\
& T_{1}^{*}=e^{-m_{1} \tau_{1}} \frac{(1-\xi) c d}{b((1-\varepsilon) k+d \beta)\left[e^{-\left(m_{1} \tau_{1}+m_{2} \tau_{2}\right)}(1-\xi) N_{T}+e^{-\left(m_{1} \tau_{1}+m_{3} \tau_{3}\right)} \xi N_{C}\right]}\left(R_{0}-1\right), \\
& C_{1}^{*}=e^{-m_{1} \tau_{1}} \frac{\xi c d}{a((1-\varepsilon) k+d \beta)\left[e^{-\left(m_{1} \tau_{1}+m_{2} \tau_{2}\right)}(1-\xi) N_{T}+e^{-\left(m_{1} \tau_{1}+m_{3} \tau_{3}\right)} \xi N_{C}\right]}\left(R_{0}-1\right), \\
& V_{1}=\frac{d}{(1-\varepsilon) k+d \beta}\left(R_{0}-1\right), \\
& T_{2}=\frac{\pi(g+\beta \mu)}{g d+(1-\varepsilon) k \mu+d \beta \mu}, \\
& T_{2}^{*}=e^{-m_{1} \tau_{1}} \frac{(1-\xi)(1-\varepsilon) k \pi \mu}{b(g d+(1-\varepsilon) k \mu+d \beta \mu)}, \\
& C_{2}^{*}=e^{-m_{1} \tau_{1}} \frac{\xi(1-\varepsilon) k \pi \mu}{a(g d+(1-\varepsilon) k \mu+d \beta \mu)} \\
& V_{2}=\frac{\mu}{g}, \\
& Z_{2}=\frac{c}{r}\left(\frac{d g R_{0}}{d g+(1-\varepsilon) k \mu+d \beta \mu}-1\right) .
\end{aligned}
$$

We note that $T_{1}, T_{1}^{*}, C_{1}^{*}$, and $V_{1}$ are positive when $R_{0}>1$. And $T_{2}, T_{2}^{*}, C_{2}^{*}, V_{2}>0$ and $Z_{2}>0$ when $d g R_{0} /(d g+(1-\varepsilon) k \mu)>1$. Now, we define another threshold parameter $R_{1}$ as

$$
R_{1}=\frac{R_{0}}{1+((1-\varepsilon) k \mu+d \beta \mu) / d g} .
$$

Clearly, $R_{0}>R_{1}$. From above, we have the following result.

Lemma 6. For system (37), one has the following:

(i) If $R_{0} \leq 1$, then the system has only one positive steady state $S_{0}$. (ii) If $R_{1} \leq 1<R_{0}$, then the system has two positive steady states $S_{0}$ and $S_{1}$.

(iii) If $R_{1}>1$, then the system has three positive steady states $S_{0}, S_{1}$, and $S_{2}$.

3.2. Global Stability Analysis. In this subsection, we investigate the global stability of system (37) by constructing suitable Lyapunov functionals and applying LaSalle invariance principle.

Theorem 7. For system (37), if $R_{0} \leq 1$, then $S_{0}$ is GAS. 
Proof. Define

$$
\begin{aligned}
U_{0}= & T_{0} H\left(\frac{T}{T_{0}}\right)+\eta_{1} T^{*}+\eta_{2} C^{*}+\eta_{3} V+\eta_{4} Z \\
& +\eta_{5} \int_{0}^{\tau_{1}} \frac{T(t-\theta) V(t-\theta)}{1+\beta V(t-\theta)} d \theta \\
& +\eta_{6} \int_{0}^{\tau_{2}} T^{*}(t-\theta) d \theta+\eta_{7} \int_{0}^{\tau_{3}} C^{*}(t-\theta) d \theta .
\end{aligned}
$$

Calculating the derivative of $U_{0}$ along the solutions of system (37), we obtain

$$
\begin{aligned}
& \frac{d U_{0}}{d t}=\left(1-\frac{T_{0}}{T}\right)\left(\pi-\frac{(1-\varepsilon) k T V}{1+\beta V}-d T\right) \\
& +\eta_{1}\left(e^{-m_{1} \tau_{1}} \frac{(1-\xi)(1-\varepsilon) k T\left(t-\tau_{1}\right) V\left(t-\tau_{1}\right)}{1+\beta V\left(t-\tau_{1}\right)}\right. \\
& \left.-b T^{*}\right) \\
& +\eta_{2}\left(e^{-m_{1} \tau_{1}} \frac{\xi(1-\varepsilon) k T\left(t-\tau_{1}\right) V\left(t-\tau_{1}\right)}{1+\beta V\left(t-\tau_{1}\right)}\right. \\
& \left.-a C^{*}\right)+\eta_{3}\left(e^{-m_{2} \tau_{2}} N_{T} b T^{*}\left(t-\tau_{2}\right)\right. \\
& \left.+e^{-m_{3} \tau_{3}} N_{C} a C^{*}\left(t-\tau_{3}\right)-r V Z-c V\right)+\eta_{4}(g V Z \\
& -\mu Z)+\eta_{5} \frac{T V}{1+\beta V}-\eta_{5} \frac{T\left(t-\tau_{1}\right) V\left(t-\tau_{1}\right)}{1+\beta V\left(t-\tau_{1}\right)} \\
& +\eta_{6} T^{*}-\eta_{6} T^{*}\left(t-\tau_{2}\right)+\eta_{7} C^{*}-\eta_{7} C^{*}\left(t-\tau_{3}\right) .
\end{aligned}
$$

Collecting terms of (42), we obtain

$$
\begin{aligned}
\frac{d U_{0}}{d t}= & \left(1-\frac{T_{0}}{T}\right)(\pi-d T)+\frac{(1-\varepsilon) k T_{0} V}{1+\beta V}-\eta_{3} c V \\
& -\eta_{4} \mu Z \\
= & -\left[d \frac{\left(T-T_{0}\right)^{2}}{T}+\eta_{3} \frac{c \beta R_{0} V^{2}}{1+\beta V}+\eta_{4} \mu Z\right] \\
& +c \eta_{3}\left(R_{0}-1\right) V .
\end{aligned}
$$

It follows that $S_{0}$ is GAS when $R_{0} \leq 1$.

Theorem 8. For system (37), assume that $R_{1} \leq 1<R_{0}$; then, $S_{1}$ is GAS.
Proof. Consider

$$
\begin{aligned}
U_{1} & =T_{1} H\left(\frac{T}{T_{1}}\right)+\eta_{1} T_{1}^{*} H\left(\frac{T^{*}}{T_{1}^{*}}\right)+\eta_{2} C_{1}^{*} H\left(\frac{C^{*}}{C_{1}^{*}}\right) \\
& +\eta_{3} V_{1} H\left(\frac{V}{V_{1}}\right)+\eta_{4} Z+\eta_{5} \frac{T_{1} V_{1}}{1+\beta V_{1}} \\
& \cdot \int_{0}^{\tau_{1}} H\left(\frac{T(t-\theta) V(t-\theta)\left(1+\beta V_{1}\right)}{T_{1} V_{1}(1+\beta V(t-\theta))}\right) d \theta \\
& +\eta_{6} T_{1}^{*} \int_{0}^{\tau_{2}} H\left(\frac{T^{*}(t-\theta)}{T_{1}^{*}}\right) d \theta \\
& +\eta_{7} C_{1}^{*} \int_{0}^{\tau_{3}} H\left(\frac{C^{*}(t-\theta)}{C_{1}^{*}}\right) d \theta .
\end{aligned}
$$

Then, $d U_{1} / d t$ is given by

$$
\begin{aligned}
& \frac{d U_{1}}{d t}=\left(1-\frac{T_{1}}{T}\right)\left(\pi-d T-\frac{(1-\varepsilon) k T V}{(1+\beta V)}\right)+\eta_{1}(1 \\
& \left.-\frac{T_{1}^{*}}{T^{*}}\right) \\
& \cdot\left(e^{-m_{1} \tau_{1}} \frac{(1-\xi)(1-\varepsilon) k T\left(t-\tau_{1}\right) V\left(t-\tau_{1}\right)}{1+\beta V\left(t-\tau_{1}\right)}\right. \\
& \left.-b T^{*}\right)+\eta_{2}\left(1-\frac{C_{1}^{*}}{C^{*}}\right) \\
& \cdot\left(e^{-m_{1} \tau_{1}} \frac{\xi(1-\varepsilon) k T\left(t-\tau_{1}\right) V\left(t-\tau_{1}\right)}{1+\beta V\left(t-\tau_{1}\right)}\right. \\
& \left.-a C^{*}\right)+\eta_{3}\left(1-\frac{V_{1}}{V}\right)\left(e^{-m_{2} \tau_{2}} N_{T} b T^{*}\left(t-\tau_{2}\right)\right. \\
& \left.+e^{-m_{3} \tau_{3}} N_{C} a C^{*}\left(t-\tau_{3}\right)-r V Z-c V\right)+\eta_{4}(g V Z \\
& -\mu Z)+\eta_{5}\left[\frac{T V}{1+\beta V}-\frac{T\left(t-\tau_{1}\right) V\left(t-\tau_{1}\right)}{\left(1+\beta V\left(t-\tau_{1}\right)\right)}\right. \\
& \left.+\frac{T_{1} V_{1}}{1+\beta V_{1}} \ln \left(\frac{T\left(t-\tau_{1}\right) V\left(t-\tau_{1}\right)(1+\beta V)}{T V\left(1+\beta V\left(t-\tau_{1}\right)\right)}\right)\right] \\
& +\eta_{6}\left[T^{*}-T^{*}\left(t-\tau_{2}\right)+T_{1}^{*} \ln \left(\frac{T^{*}\left(t-\tau_{2}\right)}{T^{*}}\right)\right] \\
& +\eta_{7}\left[C^{*}-C^{*}\left(t-\tau_{3}\right)+C_{1}^{*} \ln \left(\frac{C^{*}\left(t-\tau_{3}\right)}{C^{*}}\right)\right] \text {. }
\end{aligned}
$$

Simplifying (45), we get

$$
\begin{gathered}
\frac{d U_{1}}{d t}=\left(1-\frac{T_{1}}{T}\right)(\pi-d T)+\frac{(1-\varepsilon) k T_{1} V}{1+\beta V}-e^{m_{1} \tau_{1}} \eta_{1} \\
\cdot \frac{(1-\xi)(1-\varepsilon) k T\left(t-\tau_{1}\right) V\left(t-\tau_{1}\right)}{1+\beta V\left(t-\tau_{1}\right)} \frac{T_{1}^{*}}{T^{*}}
\end{gathered}
$$




$$
\begin{aligned}
+ & \eta_{1} b T_{1}^{*}-e^{-m_{1} \tau_{1}} \eta_{2} \\
& \cdot \frac{\xi(1-\varepsilon) k T\left(t-\tau_{1}\right) V\left(t-\tau_{1}\right)}{1+\beta V\left(t-\tau_{1}\right)} \frac{C_{1}^{*}}{C^{*}}+\eta_{2} a C_{1}^{*} \\
- & b \eta_{1} \frac{T^{*}\left(t-\tau_{2}\right) V_{1}}{V}-a \eta_{2} \frac{C^{*}\left(t-\tau_{3}\right) V_{1}}{V} \\
- & c \eta_{3} V+c \eta_{3} V_{1}+r \eta_{3} V_{1} Z-\mu \eta_{4} Z+\eta_{5} \\
& \cdot \frac{T_{1} V_{1}}{\left(1+\beta V_{1}\right)} \\
& \cdot \ln \left(\frac{T\left(t-\tau_{1}\right) V\left(t-\tau_{1}\right)(1+\beta V)}{T V\left(1+\beta V\left(t-\tau_{1}\right)\right)}\right) \\
+ & \eta_{1} b T_{1}^{*} \ln \left(\frac{T^{*}\left(t-\tau_{2}\right)}{T^{*}}\right)+\eta_{2} a C_{1}^{*} \\
& \cdot \ln \left(\frac{C^{*}\left(t-\tau_{3}\right)}{C^{*}}\right) .
\end{aligned}
$$

Applying $\pi=d T_{1}+(1-\varepsilon) k T_{1} V_{1} /\left(1+\beta V_{1}\right)$, we get

$$
\begin{aligned}
& \frac{d U_{1}}{d t}=\left(1-\frac{T_{1}}{T}\right)\left(d T_{1}-d T\right) \\
& +\frac{(1-\varepsilon) k T_{1} V_{1}}{1+\beta V_{1}}\left(1-\frac{T_{1}}{T}\right)+\frac{(1-\varepsilon) k T_{1} V}{1+\beta V} \\
& -e^{-m_{1} \tau_{1}} \eta_{1} \\
& \cdot \frac{(1-\xi)(1-\varepsilon) k T\left(t-\tau_{1}\right) V\left(t-\tau_{1}\right)}{1+\beta V\left(t-\tau_{1}\right)} \frac{T_{1}^{*}}{T^{*}} \\
& +\eta_{1} b T_{1}^{*}-e^{-m_{1} \tau_{1}} \eta_{2} \\
& \text {. } \frac{\xi(1-\varepsilon) k T\left(t-\tau_{1}\right) V\left(t-\tau_{1}\right)}{1+\beta V\left(t-\tau_{1}\right)} \frac{C_{1}^{*}}{C^{*}}+\eta_{2} a C_{1}^{*} \\
& -b \eta_{1} \frac{T^{*}\left(t-\tau_{2}\right) V_{1}}{V}-a \eta_{2} \frac{C^{*}\left(t-\tau_{3}\right) V_{1}}{V} \\
& -c \eta_{3} V+c \eta_{3} V_{1}+r \eta_{3} V_{1} Z-\mu \eta_{4} Z \\
& +\frac{T_{1} V_{1} e^{-m_{1} \tau_{1}} \eta_{1}(1-\xi)(1-\varepsilon) k}{1+\beta V_{1}} \\
& \cdot \ln \left(\frac{T\left(t-\tau_{1}\right) V\left(t-\tau_{1}\right)(1+\beta V)}{T V\left(1+\beta V\left(t-\tau_{1}\right)\right)}\right) \\
& +\frac{T_{1} V_{1} e^{-m_{1} \tau_{1}} \eta_{2} \xi(1-\varepsilon) k}{1+\beta V_{1}} \\
& \cdot \ln \left(\frac{T\left(t-\tau_{1}\right) V\left(t-\tau_{1}\right)(1+\beta V)}{T V\left(1+\beta V\left(t-\tau_{1}\right)\right)}\right) \\
& +\eta_{1} b T_{1}^{*} \ln \left(\frac{T^{*}\left(t-\tau_{2}\right)}{T^{*}}\right)+\eta_{2} a C_{1}^{*} \\
& \cdot \ln \left(\frac{C^{*}\left(t-\tau_{3}\right)}{C^{*}}\right) \text {. }
\end{aligned}
$$

$$
\begin{aligned}
c V_{1}= & e^{-m_{2} \tau_{2}} N_{T} b T_{1}^{*} \\
& +e^{-m_{3} \tau_{3}} N_{C} a C_{1}^{*},
\end{aligned}
$$

we obtain $(1-\varepsilon) k T_{1} V_{1} /\left(1+\beta V_{1}\right)=\eta_{1} b T_{1}^{*}+\eta_{2} a C_{1}^{*}$ and

$$
\begin{aligned}
\frac{d U_{1}}{d t}= & -d \frac{\left(T-T_{1}\right)^{2}}{T}+\frac{(1-\varepsilon) k T_{1} V_{1}}{1+\beta V_{1}} \frac{\left(1+\beta V_{1}\right) V}{(1+\beta V) V_{1}} \\
& +\eta_{1} b T_{1}^{*}\left(1-\frac{T_{1}}{T}\right)+\eta_{2} a C_{1}^{*}\left(1-\frac{T_{1}}{T}\right) \\
& -\eta_{1} b T_{1}^{*} \frac{T\left(t-\tau_{1}\right) V\left(t-\tau_{1}\right) T_{1}^{*}\left(1+\beta V_{1}\right)}{T_{1} V_{1} T^{*}\left(1+\beta V\left(t-\tau_{1}\right)\right)} \\
& +\eta_{1} b T_{1}^{*} \\
& -\eta_{2} a C_{1}^{*} \frac{T\left(t-\tau_{1}\right) V\left(t-\tau_{1}\right) C_{1}^{*}\left(1+\beta V_{1}\right)}{T_{1} V_{1} C^{*}\left(1+\beta V\left(t-\tau_{1}\right)\right)} \\
& +\eta_{2} a C_{1}^{*}-\eta_{1} b T_{1}^{*} \frac{V_{1} T^{*}\left(t-\tau_{2}\right)}{V T_{1}^{*}} \\
& -\eta_{2} a C_{1}^{*} \frac{C^{*}\left(t-\tau_{3}\right) V_{1}}{V C_{1}^{*}}+\eta_{1} b T_{1}^{*}+\eta_{2} a C_{1}^{*} \\
& -\frac{(1-\varepsilon) k T_{1} V_{1} \frac{V}{V_{1}}+r \eta_{3} V_{1} Z-\mu \eta_{4} Z}{1+\beta V_{1}} \\
& +\eta_{1} b T_{1}^{*} \ln \left(\frac{T\left(t-\tau_{1}\right) V\left(t-\tau_{1}\right)(1+\beta V)}{T V\left(1+\beta V\left(t-\tau_{1}\right)\right)}\right) \\
& +\eta_{2} a C_{1}^{*} \ln \left(\frac{T\left(t-\tau_{1}\right) V\left(t-\tau_{1}\right)(1+\beta V)}{T V\left(1+\beta V\left(t-\tau_{1}\right)\right)}\right) \\
& +\eta_{1} b T_{1}^{*} \ln \left(\frac{T^{*}\left(t-\tau_{2}\right)}{T^{*}}\right) \\
& +\eta_{2} a C_{1}^{*} \ln \left(\frac{C^{*}\left(t-\tau_{3}\right)}{C^{*}}\right) . \\
&
\end{aligned}
$$

Consider the following equalities:

$$
\begin{aligned}
& \ln \left(\frac{T\left(t-\tau_{1}\right) V\left(t-\tau_{1}\right)(1+\beta V)}{T V\left(1+\beta V\left(t-\tau_{1}\right)\right)}\right) \\
& \quad=\ln \left(\frac{T_{j}}{T}\right)
\end{aligned}
$$




$$
\begin{aligned}
&+\ln \left(\frac{T\left(t-\tau_{1}\right) V\left(t-\tau_{1}\right)\left(1+\beta V_{1}\right) T_{j}^{*}}{T_{j} V_{j}\left(1+\beta V\left(t-\tau_{1}\right)\right) T^{*}}\right) \\
&+\ln \left(\frac{V_{j} T^{*}}{V T_{j}^{*}}\right)+\ln \left(\frac{1+\beta V}{1+\beta V_{j}}\right), \\
& \ln \left(\frac{T\left(t-\tau_{1}\right) V\left(t-\tau_{1}\right)(1+\beta V)}{T V\left(1+\beta V\left(t-\tau_{1}\right)\right)}\right) \\
&=\ln \left(\frac{T_{j}}{T}\right) \\
&+\ln \left(\frac{T\left(t-\tau_{1}\right) V\left(t-\tau_{1}\right)\left(1+\beta V_{j}\right) C_{j}^{*}}{T_{j} V_{j}\left(1+\beta V\left(t-\tau_{1}\right)\right) C^{*}}\right) \\
&+\ln \left(\frac{V_{j} C^{*}}{V C_{j}^{*}}\right)+\ln \left(\frac{1+\beta V}{1+\beta V_{j}}\right), \\
& \ln \left(\frac{T^{*}\left(t-\tau_{2}\right)}{T^{*}}\right) \\
&=\ln \left(\frac{T^{*}\left(t-\tau_{2}\right) V_{j}}{V T_{j}^{*}}\right)+\ln \left(\frac{V T_{j}^{*}}{V_{j} T^{*}}\right), \\
& \ln \left(\frac{C^{*}\left(t-\tau_{3}\right)}{C^{*}}\right) \\
& \ln \left(\frac{C^{*}\left(t-\tau_{3}\right) V_{j}}{V C_{j}^{*}}\right)+\ln \left(\frac{V C_{j}^{*}}{V_{j} C^{*}}\right),
\end{aligned}
$$

Using (50), when $j=1$, we get

$$
\begin{aligned}
& \frac{d U_{1}}{d t}=-d \frac{\left(T-T_{1}\right)^{2}}{T}+\frac{(1-\varepsilon) k T_{1} V_{1}}{1+\beta V_{1}}\left[\frac{\left(1+\beta V_{1}\right) V}{(1+\beta V) V_{1}}\right. \\
& \left.-\frac{V}{V_{1}}-1+\frac{1+\beta V}{1+\beta V_{1}}\right]-\eta_{1} b T_{1}^{*}\left[\frac{T_{1}}{T}-1\right. \\
& \left.-\ln \left(\frac{T_{1}}{T}\right)\right]-\eta_{2} a C_{1}^{*}\left[\frac{T_{1}}{T}-1-\ln \left(\frac{T_{1}}{T}\right)\right] \\
& -\eta_{1} b T_{1}^{*}\left[\frac{T\left(t-\tau_{1}\right) V\left(t-\tau_{1}\right)\left(1+\beta V_{1}\right) T_{1}^{*}}{T_{1} V_{1}\left(1+\beta V\left(t-\tau_{1}\right)\right) T^{*}}-1\right. \\
& \left.-\ln \left(\frac{T\left(t-\tau_{1}\right) V\left(t-\tau_{1}\right)\left(1+\beta V_{1}\right) T_{1}^{*}}{T_{1} V_{1}\left(1+\beta V\left(t-\tau_{1}\right)\right) T^{*}}\right)\right] \\
& -\eta_{2} a C_{1}^{*}\left[\frac{T\left(t-\tau_{1}\right) V\left(t-\tau_{1}\right)\left(1+\beta V_{1}\right) C_{1}^{*}}{T_{1} V_{1}\left(1+\beta V\left(t-\tau_{1}\right)\right) C^{*}}-1\right. \\
& \left.-\ln \left(\frac{T\left(t-\tau_{1}\right) V\left(t-\tau_{1}\right)\left(1+\beta V_{1}\right) C_{1}^{*}}{T_{1} V_{1}\left(1+\beta V\left(t-\tau_{1}\right)\right) C^{*}}\right)\right]
\end{aligned}
$$

$$
\begin{aligned}
& -\eta_{1} b T_{1}^{*}\left[\frac{T^{*}\left(t-\tau_{2}\right) V_{1}}{V T_{1}^{*}}-1\right. \\
& \left.-\ln \left(\frac{T^{*}\left(t-\tau_{2}\right) V_{1}}{V T_{1}^{*}}\right)\right]-\eta_{2} a C_{1}^{*}\left[\frac{C^{*}\left(t-\tau_{3}\right) V_{1}}{V C_{1}^{*}}\right. \\
& \left.-1-\ln \left(\frac{C^{*}\left(t-\tau_{3}\right) V_{1}}{V C_{1}^{*}}\right)\right]-\eta_{1} b T_{1}^{*}\left[\frac{1+\beta V}{1+\beta V_{1}}\right. \\
& \left.-1-\ln \left(\frac{1+\beta V}{1+\beta V_{1}}\right)\right]-\eta_{2} a C_{1}^{*}\left[\frac{1+\beta V}{1+\beta V_{1}}-1\right. \\
& \left.-\ln \left(\frac{1+\beta V}{1+\beta V_{1}}\right)\right]+r \eta_{3}\left(V_{1}-\frac{\mu}{g}\right) Z \\
& =-d \frac{\left(T-T_{1}\right)^{2}}{T} \\
& -\frac{(1-\varepsilon) k T_{1} V_{1}}{1+\beta V_{1}}\left[\frac{\beta\left(V-V_{1}\right)^{2}}{\left(1+\beta V_{1}\right)(1+\beta V) V_{1}}\right] \\
& -\eta_{1} b T_{1}^{*}\left[H\left(\frac{T_{1}}{T}\right)\right. \\
& +H\left(\frac{T\left(t-\tau_{1}\right) V\left(t-\tau_{1}\right)\left(1+\beta V_{1}\right) T_{1}^{*}}{T_{1} V_{1}\left(1+\beta V\left(t-\tau_{1}\right)\right) T^{*}}\right) \\
& \left.+H\left(\frac{T^{*}\left(t-\tau_{2}\right) V_{1}}{V T_{1}^{*}}\right)+H\left(\frac{1+\beta V}{1+\beta V_{1}}\right)\right] \\
& -\eta_{2} a C_{1}^{*}\left[H\left(\frac{T_{1}}{T}\right)\right. \\
& +H\left(\frac{T\left(t-\tau_{1}\right) V\left(t-\tau_{1}\right)\left(1+\beta V_{1}\right) C_{1}^{*}}{T_{1} V_{1}\left(1+\beta V\left(t-\tau_{1}\right)\right) C^{*}}\right) \\
& \left.+H\left(\frac{C^{*}\left(t-\tau_{3}\right) V_{1}}{V C_{1}^{*}}\right)+H\left(\frac{1+\beta V}{1+\beta V_{1}}\right)\right] \\
& +r \eta_{3}\left(\frac{d g+\mu(1-\varepsilon) k+d \beta \mu}{g(1-\varepsilon) k+d \beta g}\right)\left[R_{1}-1\right] Z .
\end{aligned}
$$

We have if $R_{1} \leq 1<R_{0}$, then $d U_{1} / d t \leq 0$, where equality occurs at $S_{1}$. LIP implies global stability of $S_{1}$.

Theorem 9. For system (37), assume that $R_{1}>1$; then, $S_{2}$ is GAS.

Proof. We consider

$$
\begin{gathered}
U_{2}=T_{2} H\left(\frac{T}{T_{2}}\right)+\eta_{1} T_{2}^{*} H\left(\frac{T^{*}}{T_{2}^{*}}\right)+\eta_{2} C_{2}^{*} H\left(\frac{C^{*}}{C_{2}^{*}}\right) \\
+\eta_{3} V_{2} H\left(\frac{V}{V_{2}}\right)+\eta_{4} Z_{2} H\left(\frac{Z}{Z_{2}}\right)+\eta_{5} \frac{T_{2} V_{2}}{1+\beta V_{2}} \\
\cdot \int_{0}^{\tau_{1}} H\left(\frac{T(t-\theta) V(t-\theta)\left(1+\beta V_{2}\right)}{T_{2} V_{2}(1+\beta V(t-\theta))}\right) d \theta
\end{gathered}
$$




$$
\begin{aligned}
& +\eta_{6} T_{2}^{*} \int_{0}^{\tau_{2}} H\left(\frac{T^{*}(t-\theta)}{T_{2}^{*}}\right) d \theta \\
& +\eta_{7} C_{2}^{*} \int_{0}^{\tau_{3}} H\left(\frac{C^{*}(t-\theta)}{C_{2}^{*}}\right) d \theta .
\end{aligned}
$$

Calculate $d U_{2} / d t$ along the trajectories of system (37) as

$$
\begin{aligned}
& \frac{d U_{2}}{d t}=\left(1-\frac{T_{2}}{T}\right)\left(\pi-d T-\frac{(1-\varepsilon) k T V}{1+\beta V}\right)+\eta_{1}(1 \\
& \left.-\frac{T_{2}^{*}}{T^{*}}\right) \\
& \cdot\left(\frac{e^{-m_{1} \tau_{1}}(1-\xi)(1-\varepsilon) k T\left(t-\tau_{1}\right) V\left(t-\tau_{1}\right)}{1+\beta V\left(t-\tau_{1}\right)}\right. \\
& \left.-b T^{*}\right)+\eta_{2}\left(1-\frac{C_{2}^{*}}{C^{*}}\right) \\
& \cdot\left(\frac{e^{-m_{1} \tau_{1}} \xi(1-\varepsilon) k T\left(t-\tau_{1}\right) V\left(t-\tau_{1}\right)}{1+\beta V\left(t-\tau_{1}\right)}\right. \\
& \left.-a C^{*}\right)+\eta_{3}\left(1-\frac{V_{2}}{V}\right)\left(e^{-m_{2} \tau_{2}} N_{T} b T^{*}\left(t-\tau_{2}\right)\right. \\
& \left.+e^{-m_{3} \tau_{3}} N_{C} a C^{*}\left(t-\tau_{3}\right)-r V Z-c V\right)+\eta_{4}(1 \\
& \left.-\frac{Z_{2}}{Z}\right)(g V Z-\mu Z)+\eta_{5}\left[\frac{T V}{1+\beta V}\right. \\
& +\frac{T\left(t-\tau_{1}\right) V\left(t-\tau_{1}\right)}{1+\beta V\left(t-\tau_{1}\right)} \\
& +\eta_{7}\left[C^{*}-C^{*}\left(t-\tau_{3}\right)+C_{2}^{*} \ln \left(\frac{C^{*}\left(t-\tau_{3}\right)}{C^{*}}\right)\right] . \\
& +\eta_{6}\left[T^{*}-T^{*}\left(t-\tau_{2}\right)+T_{2}^{*} \ln \left(\frac{T^{*}\left(t-\tau_{2}\right)}{T^{*}}\right)\right] \\
& \left.\left.+\frac{T\left(t-\tau_{1}\right) V\left(t-\tau_{1}\right)(1+\beta V)}{T V\left(1+\beta V\left(t-\tau_{1}\right)\right)}\right)\right]
\end{aligned}
$$

Using the steady state conditions for $S_{2}$,

$$
\begin{aligned}
\pi & =d T_{2}+\frac{(1-\varepsilon) k T_{2} V_{2}}{1+\beta V_{2}}, \\
\frac{(1-\xi)(1-\varepsilon) k T_{2} V_{2}}{1+\beta V_{2}} & =e^{m_{1} \tau_{1}} b T_{2}^{*}, \\
\frac{\xi(1-\varepsilon) k T_{2} V_{2}}{1+\beta V_{2}} & =e^{m_{1} \tau_{1}} a C_{2}^{*}, \\
c V_{2}= & e^{-m_{2} \tau_{2}} N_{T} b T_{2}^{*}+e^{-m_{3} \tau_{3}} N_{C} a C_{2}^{*} \\
& -r V_{2} Z_{2},
\end{aligned}
$$

we get $(1-\varepsilon) k T_{2} V_{2} /\left(1+\beta V_{2}\right)=\eta_{1} b T_{2}^{*}+\eta_{2} a C_{2}^{*}$, and

$$
\begin{aligned}
\frac{d U_{2}}{d t}= & -d \frac{\left(T-T_{2}\right)^{2}}{T}+\frac{(1-\varepsilon) k T_{2} V_{2}}{1+\beta V_{2}} \frac{\left(1+\beta V_{2}\right) V}{(1+\beta V) V_{2}} \\
& +\eta_{1} b T_{2}^{*}\left(1-\frac{T_{2}}{T}\right)+\eta_{2} a C_{2}^{*}\left(1-\frac{T_{2}}{T}\right) \\
& -\eta_{1} b T_{2}^{*} \frac{T\left(t-\tau_{1}\right) V\left(t-\tau_{1}\right) T_{2}^{*}\left(1+\beta V_{2}\right)}{T_{2} V_{2} T^{*}\left(1+\beta V\left(t-\tau_{1}\right)\right)} \\
& +\eta_{1} b T_{2}^{*} \\
& -\eta_{2} a C_{2}^{*} \frac{T\left(t-\tau_{1}\right) V\left(t-\tau_{1}\right) C_{2}^{*}\left(1+\beta V_{2}\right)}{T_{2} V_{2} C^{*}\left(1+\beta V\left(t-\tau_{1}\right)\right)} \\
& +\eta_{2} a C_{2}^{*}-\eta_{1} b T_{2}^{*} \frac{T^{*}\left(t-\tau_{2}\right) V_{2}}{V T_{2}^{*}} \\
& -\eta_{2} a C_{2}^{*} \frac{C^{*}\left(t-\tau_{3}\right) V_{2}}{V C_{2}^{*}}+\eta_{1} b T_{2}^{*}+\eta_{2} a C_{2}^{*} \\
& -\frac{(1-\varepsilon) k T_{2} V_{2} \frac{V}{V_{2}}}{1+\beta V_{2}} \\
& +\eta_{1} b T_{2}^{*} \ln \left(\frac{T\left(t-\tau_{1}\right) V\left(t-\tau_{1}\right)(1+\beta V)}{T V\left(1+\beta V\left(t-\tau_{1}\right)\right)}\right) \\
& +\eta_{2} a C_{2}^{*} \ln \left(\frac{T\left(t-\tau_{1}\right) V\left(t-\tau_{1}\right)(1+\beta V)}{T V\left(1+\beta V\left(t-\tau_{1}\right)\right)}\right) \\
& +\eta_{1} b T_{2}^{*} \ln \left(\frac{T^{*}\left(t-\tau_{2}\right)}{T^{*}}\right) \\
& \eta_{2}^{*} \ln \left(\frac{C^{*}\left(t-\tau_{3}\right)}{C^{*}}\right) .
\end{aligned}
$$

Using (50), when $j=2$, we get

$$
\begin{aligned}
& \frac{d U_{2}}{d t}=-d \frac{\left(T-T_{2}\right)^{2}}{T}+\frac{(1-\varepsilon) k T_{2} V_{2}}{1+\beta V_{2}}\left[\frac{\left(1+\beta V_{2}\right) V}{(1+\beta V) V_{2}}\right. \\
& \left.-\frac{V}{V_{2}}-1+\frac{1+\beta V}{1+\beta V_{2}}\right]-\eta_{1} b T_{2}^{*}\left[\frac{T_{2}}{T}-1\right. \\
& \left.-\ln \left(\frac{T_{2}}{T}\right)\right]-\eta_{2} a C_{2}^{*}\left[\frac{T_{2}}{T}-1-\ln \left(\frac{T_{2}}{T}\right)\right] \\
& -\eta_{1} b T_{2}^{*}\left[\frac{T\left(t-\tau_{1}\right) V\left(t-\tau_{1}\right)(1+\beta V) T_{2}^{*}}{T_{2} V_{2} T^{*}\left(1+\beta V\left(t-\tau_{1}\right)\right)}-1\right. \\
& \left.-\ln \left(\frac{T\left(t-\tau_{1}\right) V\left(t-\tau_{1}\right)(1+\beta V) T_{2}^{*}}{T_{2} V_{2} T^{*}\left(1+\beta V\left(t-\tau_{1}\right)\right)}\right)\right] \\
& -\eta_{2} a C_{2}^{*}\left[\frac{T\left(t-\tau_{1}\right) V\left(t-\tau_{1}\right)(1+\beta V) C_{2}^{*}}{T_{2} V_{2} C^{*}\left(1+\beta V\left(t-\tau_{1}\right)\right)}-1\right. \\
& \left.-\ln \left(\frac{T\left(t-\tau_{1}\right) V\left(t-\tau_{1}\right)(1+\beta V) C_{2}^{*}}{T_{2} V_{2} C^{*}\left(1+\beta V\left(t-\tau_{1}\right)\right)}\right)\right]
\end{aligned}
$$




$$
\begin{aligned}
& -\eta_{1} b T_{2}^{*}\left[\frac{T^{*}\left(t-\tau_{2}\right) V_{2}}{V T_{2}^{*}}-1\right. \\
& \left.-\ln \left(\frac{T^{*}\left(t-\tau_{2}\right) V_{2}}{V T_{2}^{*}}\right)\right]-\eta_{2} a C_{2}^{*}\left[\frac{C^{*}\left(t-\tau_{3}\right) V_{2}}{V C_{2}^{*}}\right. \\
& \left.-1-\ln \left(\frac{C^{*}\left(t-\tau_{3}\right) V_{2}}{V C_{2}^{*}}\right)\right]-\eta_{1} b T_{2}^{*}\left[\frac{1+\beta V}{1+\beta V_{2}}\right. \\
& \left.-1-\ln \left(\frac{1+\beta V}{1+\beta V_{2}}\right)\right]-\eta_{2} a C_{2}^{*}\left[\frac{1+\beta V}{1+\beta V_{2}}-1\right. \\
& \left.-\ln \left(\frac{1+\beta V}{1+\beta V_{2}}\right)\right] \\
& =-d \frac{\left(T-T_{2}\right)^{2}}{T} \\
& -\frac{(1-\varepsilon) k T_{2} V_{2}}{1+\beta V_{2}}\left[\frac{\beta\left(V-V_{2}\right)^{2}}{\left(1+\beta V_{2}\right)(1+\beta V) V_{2}}\right] \\
& -\eta_{1} b T_{2}^{*}\left[H\left(\frac{T_{2}}{T}\right)\right. \\
& +H\left(\frac{T\left(t-\tau_{1}\right) V\left(t-\tau_{1}\right)(1+\beta V) T_{2}^{*}}{T_{2} V_{2} T^{*}\left(1+\beta V\left(t-\tau_{1}\right)\right)}\right) \\
& \left.+H\left(\frac{T^{*}\left(t-\tau_{2}\right) V_{2}}{V T_{2}^{*}}\right)+H\left(\frac{1+\beta V}{1+\beta V_{2}}\right)\right] \\
& -\eta_{2} a C_{2}^{*}\left[H\left(\frac{T_{2}}{T}\right)\right. \\
& +H\left(\frac{T\left(t-\tau_{1}\right) V\left(t-\tau_{1}\right)(1+\beta V) C_{2}^{*}}{T_{2} V_{2} C^{*}\left(1+\beta V\left(t-\tau_{1}\right)\right)}\right) \\
& \left.+H\left(\frac{C^{*}\left(t-\tau_{3}\right) V_{2}}{V C_{2}^{*}}\right)+H\left(\frac{1+\beta V}{1+\beta V_{2}}\right)\right] \text {. }
\end{aligned}
$$

Similar to the proof of Theorem 5, one can show that $S_{2}$ is GAS.

\section{Model with General Incidence}

In the following model, we assume the incidence rate is given by a general function of the concentration of $\mathrm{CD} 4^{+} \mathrm{T}$ cells and viruses:

$$
\begin{aligned}
\dot{T}(t)= & \pi-\Phi(T(t), V(t))-d T, \\
\dot{T}^{*}(t)= & e^{-m_{1} \tau_{1}}(1-\xi) \Phi\left(T\left(t-\tau_{1}\right), V\left(t-\tau_{1}\right)\right) \\
& -b T^{*}(t), \\
\dot{C}^{*}(t)= & e^{-m_{1} \tau_{1}} \xi \Phi\left(T\left(t-\tau_{1}\right), V\left(t-\tau_{1}\right)\right)-a C^{*}(t),
\end{aligned}
$$

$$
\begin{aligned}
\dot{V}(t)= & e^{-m_{2} \tau_{2}} N_{T} b T^{*}\left(t-\tau_{2}\right) \\
& +e^{-m_{3} \tau_{3}} N_{C} a C^{*}\left(t-\tau_{3}\right)-r V(t) Z(t) \\
& -c V(t), \\
\dot{Z}(t)= & g V(t) Z(t)-\mu Z(t) .
\end{aligned}
$$

The incidence rate is given by $\Phi(T(t), V(t))$ which is assumed to be continuously differentiable; moreover, it satisfies the following conditions.

Condition C1. (i) Consider $\Phi(T, V)>0$ and $\Phi(0, V)=$ $\Phi(T, 0)=0$, for all $T, V \in(0, \infty)$. (ii) Consider $\partial \Phi(T, V) / \partial T>0, \partial \Phi(T, V) / \partial V>0$, and $\partial \Phi(T, 0) / \partial V>0$, for all $T, V \in(0, \infty)$.

Condition C2. (i) Consider $\Phi(T, V) \leqslant V(\partial \Phi(T, 0) / \partial V)$, for all $T, V \in(0, \infty)$. (ii) Consider $(d / d T)(\partial \Phi(T, 0) / \partial V)>0$.

The nonnegativity and boundedness of the solutions of model (57)-(61) can be shown as given in Section 2 .

\subsection{Steady States}

Lemma 10. Suppose that Conditions $C 1$ and $C 2$ are satisfied; then, there exist two bifurcation parameters $R_{0}$ and $R_{1}$ with $R_{0}>R_{1}>0$. Moreover,

(i) if $R_{0} \leq 1$, then the system has only one positive steady state $S_{0}$,

(ii) if $R_{1} \leq 1<R_{0}$, then the system has two positive steady states $S_{0}$ and $S_{1}$,

(iii) if $R_{1}>1$, then the system has three positive steady states $S_{0}, S_{1}$, and $S_{2}$.

Proof. Let

$$
\begin{aligned}
\pi-\Phi(T, V)-d T & =0, \\
e^{-m_{1} \tau_{1}}(1-\xi) \Phi(T, V)-b T^{*} & =0, \\
e^{-m_{1} \tau_{1}} \xi \Phi(T, V)-a C^{*} & =0, \\
e^{-m_{2} \tau_{2}} N_{T} b T^{*}+e^{-m_{3} \tau_{3}} N_{C} a C^{*}-r V Z-c V & =0, \\
g V Z-\mu Z & =0 .
\end{aligned}
$$

Equation (66) admits two solutions, $Z=0$ and $V=\mu / g$. Substituting $Z=0$ into (63) and (64), we obtain $T^{*}$ and $C^{*}$ as

$$
\begin{aligned}
T^{*} & =\frac{e^{-m_{1} \tau_{1}}(1-\xi) \Phi(T, V)}{b}, \\
C^{*} & =\frac{e^{-m_{1} \tau_{1}} \xi \Phi(T, V)}{a} .
\end{aligned}
$$

Substituting (67) into (65), we get

$$
\begin{aligned}
& {\left[e^{-\left(m_{1} \tau_{1}+m_{2} \tau_{2}\right)}(1-\xi) N_{T}+e^{-\left(m_{1} \tau_{1}+m_{3} \tau_{3}\right)} \xi N_{C}\right] \Phi(T, V)} \\
& -c V=0 .
\end{aligned}
$$


Using Condition C1, we have $V=0$ is a solution of (68). Therefore, $T^{*}=C^{*}=0$ and $T=T_{0}$ which leads to $S_{0}=$ $\left(T_{0}, 0,0,0,0\right)$. If $V \neq 0$, then, from (62) and (68), we obtain

$$
V=\frac{\gamma \Phi(T, V)}{c}=\frac{\gamma(\pi-d T)}{c} \Longrightarrow T=T_{0}-\frac{c V}{d \gamma},
$$

where $\gamma=\left[e^{-\left(m_{1} \tau_{1}+m_{2} \tau_{2}\right)}(1-\xi) N_{T}+e^{-\left(m_{1} \tau_{1}+m_{3} \tau_{3}\right)} \xi N_{C}\right]$. Then, (68) becomes

$$
\gamma \Phi\left(T_{0}-\frac{c}{d \gamma} V, V\right)-c V=0
$$

Let us define a function $\Psi_{1}$ as

$$
\Psi_{1}(V)=\gamma \Phi\left(T_{0}-\frac{c}{d \gamma} V, V\right)-c V=0 .
$$

Condition $\mathrm{C} 1$ implies that $\Psi_{1}(0)=0$, and when $V=V^{*}=$ $\pi \gamma / c>0$, then $\Psi_{1}\left(V^{*}\right)=-c V^{*}<0$. We have

$$
\Psi_{1}^{\prime}(0)=\gamma\left[-\frac{c}{d \gamma} \frac{\partial \Phi\left(T_{0}, 0\right)}{\partial T}+\frac{\partial \Phi\left(T_{0}, 0\right)}{\partial V}\right]-c .
$$

From Condition $\mathrm{C} 1$, we have $\partial \Phi\left(T_{0}, 0\right) / \partial T=0$; then,

$$
\Psi_{1}^{\prime}(0)=\gamma \frac{\partial \Phi\left(T_{0}, 0\right)}{\partial V}-c=c\left(\frac{\gamma}{c} \frac{\partial \Phi\left(T_{0}, 0\right)}{\partial V}-1\right) .
$$

Therefore, if $\Psi_{1}^{\prime}(0)>0$, that is, $(\gamma / c)\left(\partial \Phi\left(T_{0}, 0\right) / \partial V\right)>1$, then there exists $V_{1} \in\left(0, V^{*}\right)$ such that $\Psi_{1}\left(V_{1}\right)=0$. From (62), we define a function $\Psi_{2}$ as

$$
\Psi_{2}(T)=\pi-d T-\Phi\left(T, V_{1}\right)=0 .
$$

Using Condition C1, we have $\Psi_{2}(0)=\pi>0$ and $\Psi_{2}\left(T_{0}\right)=$ $-\Phi\left(T_{0}, V_{1}\right)<0$. Since $\Psi_{2}$ is a strictly decreasing function, then there exists a unique $T_{1} \in\left(0, T_{0}\right)$ such that $\Psi_{2}\left(T_{1}\right)=$ 0 . It follows that $T_{1}^{*}=e^{-m_{1} \tau_{1}}(1-\xi) \Phi\left(T_{1}, V_{1}\right) / b>0$ and $C_{1}^{*}=e^{-m_{1} \tau_{1}} \xi \Phi\left(T_{1}, V_{1}\right) / a>0$. It means that an infected steady state without humoral immune response $S_{1}\left(T_{1}, T_{1}^{*}, C_{1}^{*}, V_{1}, 0\right)$ exists when $(\gamma / c)\left(\partial \Phi\left(T_{0}, 0\right) / \partial V\right)>1$. Now, we define the parameter $R_{0}$ as

$$
R_{0}=\frac{\gamma}{c} \frac{\partial \Phi\left(T_{0}, 0\right)}{\partial V} .
$$

The other possibility of (66) is $V_{2}=\mu / g$. Insert $V_{2}$ in (62) and define $\Psi_{3}$ as

$$
\Psi_{3}(T)=\pi-d T-\Phi\left(T, V_{2}\right)=0 .
$$

Applying Condition C1, then $\Psi_{3}$ is a strictly decreasing function. Moreover, $\Psi_{3}(0)=\pi>0$ and $\Psi_{3}\left(T_{0}\right)=$ $-\Phi\left(T_{0}, V_{2}\right)<0$. Thus, there exists a unique $T_{2} \in\left(0, T_{0}\right)$ such that $\Psi_{3}\left(T_{2}\right)=0$. It follows from (63)-(65) that

$$
\begin{aligned}
& T_{2}^{*}=\frac{e^{-m_{1} \tau_{1}}(1-\xi) \Phi\left(T_{2}, V_{2}\right)}{b}, \\
& C_{2}^{*}=\frac{e^{-m_{1} \tau_{1}} \xi \Phi\left(T_{2}, V_{2}\right)}{a}, \\
& Z_{2}=\frac{c}{r}\left[\frac{\gamma \Phi\left(T_{2}, V_{2}\right)}{c V_{2}}-1\right] .
\end{aligned}
$$

Thus, $T_{2}^{*}>0$ and $C_{2}^{*}>0$; moreover, $Z_{2}>0$ when $\gamma \Phi\left(T_{2}, V_{2}\right) / c V_{2}>1$. Now, we define parameter $R_{1}$ as

$$
R_{1}=\frac{\gamma \Phi\left(T_{2}, V_{2}\right)}{c V_{2}}
$$

Hence, $Z_{2}$ can be rewritten as $Z_{2}=(c / r)\left(R_{1}-1\right)$. It follows that $S_{2}\left(T_{2}, T_{2}^{*}, C_{2}^{*}, V_{2}, Z_{2}\right)$ exists when $R_{1}>1$.

Conditions $\mathrm{C} 1$ and $\mathrm{C} 2$ imply that

$$
\begin{aligned}
R_{1} & =\frac{\gamma \Phi\left(T_{2}, V_{2}\right)}{c V_{2}} \leqslant \frac{\gamma}{c} \frac{\partial \Phi\left(T_{2}, 0\right)}{\partial V}<\frac{\gamma}{c} \frac{\partial \Phi\left(T_{0}, 0\right)}{\partial V} \\
& =R_{0} .
\end{aligned}
$$

\subsection{Global Stability Analysis}

Theorem 11. Let Conditions C1 and C2 be satisfied and $R_{0} \leq 1$; then, $S_{0}$ for system (57)-(61) is GAS.

Proof. Define

$$
\begin{aligned}
U_{0}= & T-T_{0}-\int_{T_{0}}^{T} \lim _{V \rightarrow 0^{+}} \frac{\Phi\left(T_{0}, V\right)}{\Phi(\rho, V)} d \rho+\eta_{1} T^{*}+\eta_{2} C^{*} \\
& +\eta_{3} V+\eta_{4} Z+\int_{0}^{\tau_{1}} \Phi(T(t-\theta), V(t-\theta)) d \theta \\
& +\eta_{6} \int_{0}^{\tau_{2}} T^{*}(t-\theta) d \theta+\eta_{7} \int_{0}^{\tau_{3}} C^{*}(t-\theta) d \theta .
\end{aligned}
$$

Then,

$$
\begin{aligned}
& \frac{d U_{0}}{d t}=\left(1-\lim _{V \rightarrow 0^{+}} \frac{\Phi\left(T_{0}, V\right)}{\Phi(T, V)}\right)(\pi-d T-\Phi(T, V)) \\
& \quad+\eta_{1}\left(e^{-m_{1} \tau_{1}}(1-\xi) \Phi\left(T\left(t-\tau_{1}\right), V\left(t-\tau_{1}\right)\right)\right. \\
& \left.\quad-b T^{*}\right)+\eta_{2}\left(e^{-m_{1} \tau_{1}} \xi \Phi\left(T\left(t-\tau_{1}\right), V\left(t-\tau_{1}\right)\right)\right. \\
& \left.-a C^{*}\right)+\eta_{3}\left(e^{-m_{2} \tau_{2}} N_{T} b T^{*}\left(t-\tau_{2}\right)\right. \\
& \left.+e^{-m_{3} \tau_{3}} N_{C} a C^{*}\left(t-\tau_{3}\right)-r V Z-c V\right)+\eta_{4}(g V Z \\
& -\mu Z)+\Phi(T, V)-\Phi\left(T\left(t-\tau_{1}\right), V\left(t-\tau_{1}\right)\right) \\
& +\eta_{6} T^{*}-\eta_{6} T^{*}\left(t-\tau_{2}\right)+\eta_{7} C^{*}-\eta_{7} C^{*}\left(t-\tau_{3}\right) .
\end{aligned}
$$

Equation (81) can be simplified as

$$
\begin{aligned}
\frac{d U_{0}}{d t}= & \left(1-\lim _{V \rightarrow 0^{+}} \frac{\Phi\left(T_{0}, V\right)}{\Phi(T, V)}\right)(\pi-d T) \\
& +\Phi(T, V) \lim _{V \rightarrow 0^{+}} \frac{\Phi\left(T_{0}, V\right)}{\Phi(T, V)}-c \eta_{3} V-\eta_{4} \mu Z
\end{aligned}
$$




$$
\begin{aligned}
= & \pi\left(1-\frac{\partial \Phi\left(T_{0}, 0\right) / \partial V}{\partial \Phi(T, 0) / \partial V}\right)\left(1-\frac{T}{T_{0}}\right) \\
& +\Phi(T, V) \frac{\partial \Phi\left(T_{0}, 0\right) / \partial V}{\partial \Phi(T, 0) / \partial V}-c \eta_{3} V-\eta_{4} \mu Z \\
\leqslant & \pi\left(1-\frac{\partial \Phi\left(T_{0}, 0\right) / \partial V}{\partial \Phi(T, 0) / \partial V}\right)\left(1-\frac{T}{T_{0}}\right) \\
& +V \frac{\partial \Phi\left(T_{0}, 0\right)}{\partial V}-c \eta_{3} V-\eta_{4} \mu Z \\
= & \pi\left(1-\frac{\partial \Phi\left(T_{0}, 0\right) / \partial V}{\partial \Phi(T, 0) / \partial V}\right)\left(1-\frac{T}{T_{0}}\right) \\
& +c \eta_{3}\left(\frac{1}{c \eta_{3}} \frac{\partial \Phi\left(T_{0}, 0\right)}{\partial V}-1\right) V-\eta_{4} \mu Z \\
= & \pi\left(1-\frac{\partial \Phi\left(T_{0}, 0\right) / \partial V}{\partial \Phi(T, 0) / \partial V}\right)\left(1-\frac{T}{T_{0}}\right) \\
& +c \eta_{3}\left(R_{0}-1\right) V-\eta_{4} \mu Z .
\end{aligned}
$$

Based on Condition C2, the first term of (82) is less than or equal to zero. Therefore, if $R_{0} \leq 1$, then $d U_{0} / d t \leq 0$, for all $T, V, Z>0$. Similar to the previous sections, one can show that $S_{0}$ is GAS.

\section{Condition C3. Consider}

$$
\begin{aligned}
&\left(\frac{\Phi(T, V)}{\Phi\left(T, V_{i}\right)}-\frac{V}{V_{i}}\right)\left(1-\frac{\Phi\left(T, V_{i}\right)}{\Phi(T, V)}\right) \leqslant 0, \\
& \forall T, V \in(0, \infty), i=1,2 .
\end{aligned}
$$

Lemma 12. Suppose that $R_{0}>1$ and Conditions C1-C3 hold. Then, $T_{1}, T_{2}, T_{1}^{*}, T_{2}^{*}, C_{1}^{*}, C_{2}^{*}, V_{1}, V_{2}$ exist satisfying

$$
\operatorname{sgn}\left(R_{1}-1\right)=\operatorname{sgn}\left(T_{2}-T_{1}\right)=\operatorname{sgn}\left(V_{1}-V_{2}\right) .
$$

Proof. From Condition C1, for $T_{1}, T_{2}, V_{1}, V_{2}>0$, we have

$$
\begin{aligned}
& \left(\Phi\left(T_{2}, V_{2}\right)-\Phi\left(T_{1}, V_{2}\right)\right)\left(T_{2}-T_{1}\right)>0 . \\
& \left(\Phi\left(T_{1}, V_{2}\right)-\Phi\left(T_{1}, V_{1}\right)\right)\left(V_{2}-V_{1}\right)>0,
\end{aligned}
$$

Applying Condition C3 when $i=1, T=T_{1}$, and $V=V_{2}$, we get

$$
\begin{aligned}
& \left(\Phi\left(T_{1}, V_{2}\right) V_{1}-\Phi\left(T_{1}, V_{1}\right) V_{2}\right) \\
& \cdot\left(\Phi\left(T_{1}, V_{2}\right)-\Phi\left(T_{1}, V_{1}\right)\right) \leqslant 0 .
\end{aligned}
$$

It follows from inequality (86) that

$$
\left(\Phi\left(T_{1}, V_{2}\right) V_{1}-\Phi\left(T_{1}, V_{1}\right) V_{2}\right)\left(V_{1}-V_{2}\right)>0 .
$$

We assume that $\operatorname{sgn}\left(V_{2}-V_{1}\right)=\operatorname{sgn}\left(T_{2}-T_{1}\right)$. Then, we have

$$
\begin{aligned}
(\pi- & \left.d T_{2}\right)-\left(\pi-d T_{1}\right)=\Phi\left(T_{2}, V_{2}\right)-\Phi\left(T_{1}, V_{1}\right) \\
= & \Phi\left(T_{2}, V_{2}\right)-\Phi\left(T_{1}, V_{2}\right)+\Phi\left(T_{1}, V_{2}\right) \\
& -\Phi\left(T_{1}, V_{1}\right),
\end{aligned}
$$

and using inequalities (85) and (86), we obtain $\operatorname{sgn}\left(T_{1}-\right.$ $\left.T_{2}\right)=\operatorname{sgn}\left(T_{2}-T_{1}\right)$, and this leads to contradiction. Therefore, $\operatorname{sgn}\left(V_{1}-V_{2}\right)=\operatorname{sgn}\left(T_{2}-T_{1}\right)$. From the conditions of $S_{1}$, we have $\gamma \Phi\left(T_{1}, V_{1}\right) / c V_{1}=1$; then,

$$
\begin{gathered}
R_{1}-1=\frac{\gamma}{c}\left(\frac{1}{V_{2}}\left(\Phi\left(T_{2}, V_{2}\right)-\Phi\left(T_{1}, V_{2}\right)\right)\right. \\
\left.+\frac{1}{V_{1} V_{2}}\left(\Phi\left(T_{1}, V_{2}\right) V_{1}-\Phi\left(T_{1}, V_{1}\right) V_{2}\right)\right)
\end{gathered}
$$

Using inequalities (85) and (88), we obtain $\operatorname{sgn}\left(R_{1}-1\right)=$ $\operatorname{sgn}\left(V_{1}-V_{2}\right)$.

Theorem 13. Assume that $R_{1} \leq 1<R_{0}$ and Conditions C1-C3 are satisfied; then, $S_{1}$ for system (57)-(61) is GAS.

Proof. Let us define

$$
\begin{aligned}
& U_{1} \\
& =T-T_{1}-\int_{T_{1}}^{T} \frac{\Phi\left(T_{1}, V_{1}\right)}{\Phi\left(\rho, V_{1}\right)} d \rho+\eta_{1} T_{1}^{*} H\left(\frac{T^{*}}{T_{1}^{*}}\right) \\
& +\eta_{2} C_{1}^{*} H\left(\frac{C^{*}}{C_{1}^{*}}\right)+\eta_{3} V_{1} H\left(\frac{V}{V_{1}}\right)+\eta_{4} Z \\
& +\Phi\left(T_{1}, V_{1}\right) \int_{0}^{\tau_{1}} H\left(\frac{\Phi(T(t-\theta), V(t-\theta))}{\Phi\left(T_{1}, V_{1}\right)}\right) d \theta \\
& +\eta_{6} T_{1}^{*} \int_{0}^{\tau_{2}} H\left(\frac{T^{*}(t-\theta)}{T_{1}^{*}}\right) d \theta \\
& +\eta_{7} C_{1}^{*} \int_{0}^{\tau_{3}} H\left(\frac{C^{*}(t-\theta)}{C_{1}^{*}}\right) d \theta \text {. }
\end{aligned}
$$

Then,

$$
\begin{aligned}
& \frac{d U_{1}}{d t}=\left(1-\frac{\Phi\left(T_{1}, V_{1}\right)}{\Phi\left(T, V_{1}\right)}\right)(\pi-d T-\Phi(T, V)) \\
& +\eta_{1}\left(1-\frac{T_{1}^{*}}{T^{*}}\right) \\
& \cdot\left(e^{-m_{1} \tau_{1}}(1-\xi) \Phi\left(T\left(t-\tau_{1}\right), V\left(t-\tau_{1}\right)\right)\right. \\
& \left.-b T^{*}\right)+\eta_{2}\left(1-\frac{C_{1}^{*}}{C^{*}}\right) \\
& \cdot\left(e^{-m_{1} \tau_{1}} \xi \Phi\left(T\left(t-\tau_{1}\right), V\left(t-\tau_{1}\right)\right)\right. \\
& \left.-a C^{*}\right)+\eta_{3}\left(1-\frac{V_{1}}{V}\right)\left(e^{-m_{2} \tau_{2}} N_{T} b T^{*}\left(t-\tau_{2}\right)\right. \\
& \left.+e^{-m_{3} \tau_{3}} N_{C} a C^{*}\left(t-\tau_{3}\right)-r V Z-c V\right)+\eta_{4}(g V Z \\
& -\mu Z)+\Phi(T, V)-\Phi\left(T\left(t-\tau_{1}\right), V\left(t-\tau_{1}\right)\right)
\end{aligned}
$$




$$
\begin{aligned}
& +\Phi\left(T_{1}, V_{1}\right) \ln \frac{\Phi\left(T\left(t-\tau_{1}\right), V\left(t-\tau_{1}\right)\right)}{\Phi(T, V)}+\eta_{6}\left[T^{*}\right. \\
& \left.-T^{*}\left(t-\tau_{2}\right)+T_{1}^{*} \ln \left(\frac{T^{*}\left(t-\tau_{2}\right)}{T^{*}}\right)\right]+\eta_{7}\left[C^{*}\right. \\
& \left.-C^{*}\left(t-\tau_{3}\right)+C_{1}^{*} \ln \left(\frac{C^{*}\left(t-\tau_{3}\right)}{C^{*}}\right)\right] .
\end{aligned}
$$

Collecting terms of (92), we obtain

$$
\begin{aligned}
\frac{d U_{1}}{d t} & =\left(1-\frac{\Phi\left(T_{1}, V_{1}\right)}{\Phi\left(T, V_{1}\right)}\right)(\pi-d T) \\
& +\Phi(T, V) \frac{\Phi\left(T_{1}, V_{1}\right)}{\Phi\left(T, V_{1}\right)} \\
& -e^{-m_{1} \tau_{1}} \eta_{1}(1-\xi) \Phi\left(T\left(t-\tau_{1}\right), V\left(t-\tau_{1}\right)\right) \frac{T_{1}^{*}}{T^{*}} \\
& +\eta_{1} b T_{1}^{*} \\
& -e^{-m_{1} \tau_{1}} \eta_{2} \xi \Phi\left(T\left(t-\tau_{1}\right), V\left(t-\tau_{1}\right)\right) \frac{C_{1}^{*}}{C^{*}} \\
& +\eta_{2} a C_{1}^{*}-b \eta_{1} \frac{T^{*}\left(t-\tau_{2}\right) V_{1}}{V} \\
& -a \eta_{2} \frac{C^{*}\left(t-\tau_{3}\right) V_{1}}{V}-c \eta_{3} V+c \eta_{3} V_{1}+\eta_{3} r V_{1} Z \\
& -\eta_{4} \mu Z \\
& +\Phi\left(T_{1}, V_{1}\right) \ln \left(\frac{\Phi\left(T\left(t-\tau_{1}\right), V\left(t-\tau_{1}\right)\right)}{\Phi(T, V)}\right) \\
& +\eta_{1} b T_{1}^{*} \ln \left(\frac{T^{*}\left(t-\tau_{2}\right)}{T^{*}}\right) \\
& +\eta_{2} a C_{1}^{*} \ln \left(\frac{C^{*}\left(t-\tau_{3}\right)}{C^{*}}\right) .
\end{aligned}
$$

Applying $\pi=d T_{1}+\Phi\left(T_{1}, V_{1}\right)$, we get

$$
\begin{aligned}
\frac{d U_{1}}{d t}=( & \left.1-\frac{\Phi\left(T_{1}, V_{1}\right)}{\Phi\left(T, V_{1}\right)}\right)\left(d T_{1}-d T\right)+\Phi\left(T_{1}, V_{1}\right) \\
& \cdot\left(1-\frac{\Phi\left(T_{1}, V_{1}\right)}{\Phi\left(T, V_{1}\right)}\right)+\Phi(T, V) \frac{\Phi\left(T_{1}, V_{1}\right)}{\Phi\left(T, V_{1}\right)} \\
- & e^{-m_{1} \tau_{1}} \eta_{1}(1-\xi) \Phi\left(T\left(t-\tau_{1}\right), V\left(t-\tau_{1}\right)\right) \\
& \cdot \frac{T_{1}^{*}}{T^{*}}+\eta_{1} b T_{1}^{*} \\
- & e^{-m_{1} \tau_{1}} \eta_{2} \xi \Phi\left(T\left(t-\tau_{1}\right), V\left(t-\tau_{1}\right)\right) \frac{C_{1}^{*}}{C^{*}}
\end{aligned}
$$

$$
\begin{aligned}
+ & \eta_{2} a C_{1}^{*}-\eta_{1} b \frac{T^{*}\left(t-\tau_{2}\right) V_{1}}{V}-\eta_{2} a \\
& \cdot \frac{C^{*}\left(t-\tau_{3}\right) V_{1}}{V}-c \eta_{3} V+c \eta_{3} V_{1}+\eta_{3} r V_{1} Z \\
- & \eta_{4} \mu Z+\Phi\left(T_{1}, V_{1}\right) e^{-m_{1} \tau_{1}} \eta_{1}(1-\xi) \\
& \cdot \ln \left(\frac{\Phi\left(T\left(t-\tau_{1}\right), V\left(t-\tau_{1}\right)\right)}{\Phi(T, V)}\right) \\
+ & \Phi\left(T_{1}, V_{1}\right) e^{-m_{1} \tau_{1}} \eta_{2} \xi \\
& \cdot \ln \left(\frac{\Phi\left(T\left(t-\tau_{1}\right), V\left(t-\tau_{1}\right)\right)}{\Phi(T, V)}\right)+\eta_{1} b T_{1}^{*} \\
& \cdot \ln \left(\frac{T^{*}\left(t-\tau_{2}\right)}{T^{*}}\right)+\eta_{2} a C_{1}^{*} \\
& \cdot \ln \left(\frac{C^{*}\left(t-\tau_{3}\right)}{C^{*}}\right) .
\end{aligned}
$$

Using the steady state conditions for $S_{1}$,

$$
\begin{aligned}
(1-\xi) \Phi\left(T_{1}, V_{1}\right) & =e^{m_{1} \tau_{1}} b T_{1}^{*}, \\
\xi \Phi\left(T_{1}, V_{1}\right) & =e^{m_{1} \tau_{1}} a C_{1}^{*}, \\
c V_{1} & =e^{-m_{2} \tau_{2}} N_{T} b T_{1}^{*}+e^{-m_{3} \tau_{3}} N_{C} a C_{1}^{*},
\end{aligned}
$$

then we have $\Phi\left(T_{1}, V_{1}\right)=\eta_{1} b T_{1}^{*}+\eta_{2} a C_{1}^{*}$ and

$$
\begin{aligned}
\frac{d U_{1}}{d t}= & d T_{1}\left(1-\frac{\Phi\left(T_{1}, V_{1}\right)}{\Phi\left(T, V_{1}\right)}\right)\left(1-\frac{T}{T_{1}}\right) \\
& +\Phi\left(T_{1}, V_{1}\right) \frac{\Phi(T, V)}{\Phi\left(T, V_{1}\right)} \\
& +\eta_{1} b T_{1}^{*}\left(1-\frac{\Phi\left(T_{1}, V_{1}\right)}{\Phi\left(T, V_{1}\right)}\right) \\
& +\eta_{2} a C_{1}^{*}\left(1-\frac{\Phi\left(T_{1}, V_{1}\right)}{\Phi\left(T, V_{1}\right)}\right) \\
& -\eta_{1} b T_{1}^{*} \frac{\Phi\left(T\left(t-\tau_{1}\right), V\left(t-\tau_{1}\right)\right) T_{1}^{*}}{\Phi\left(T_{1}, V_{1}\right) T^{*}} \\
& +\eta_{1} b T_{1}^{*} \\
& -\eta_{2} a C_{1}^{*} \frac{\Phi\left(T\left(t-\tau_{1}\right), V\left(t-\tau_{1}\right)\right) C_{1}^{*}}{\Phi\left(T_{1}, V_{1}\right) C^{*}} \\
& +\eta_{2} a C_{1}^{*}-\eta_{1} b T_{1}^{*} \frac{T^{*}\left(t-\tau_{2}\right) V_{1}}{V T_{1}^{*}} \\
& -\eta_{2} a C_{1}^{*} \frac{C^{*}\left(t-\tau_{3}\right) V_{1}}{V C_{1}^{*}}+\eta_{1} b T_{1}^{*}+\eta_{2} a C_{1}^{*}
\end{aligned}
$$




$$
\begin{aligned}
& -\Phi\left(T_{1}, V_{1}\right) \frac{V}{V_{1}}+\eta_{3} r\left(V_{1}-\frac{\mu}{g}\right) Z \\
& +\eta_{1} b T_{1}^{*} \ln \left(\frac{\Phi\left(T\left(t-\tau_{1}\right), V\left(t-\tau_{1}\right)\right)}{\Phi(T, V)}\right) \\
& +\eta_{2} a C_{1}^{*} \ln \left(\frac{\Phi\left(T\left(t-\tau_{1}\right), V\left(t-\tau_{1}\right)\right)}{\Phi(T, V)}\right) \\
& +\eta_{1} b T_{1}^{*} \ln \left(\frac{T^{*}\left(t-\tau_{2}\right)}{T^{*}}\right) \\
& +\eta_{2} a C_{1}^{*} \ln \left(\frac{C^{*}\left(t-\tau_{3}\right)}{C^{*}}\right) .
\end{aligned}
$$

in case of $j=1$, we obtain

$$
\frac{d U_{1}}{d t}=d T_{1}\left(1-\frac{\Phi\left(T_{1}, V_{1}\right)}{\Phi\left(T, V_{1}\right)}\right)\left(1-\frac{T}{T_{1}}\right)+\Phi\left(T_{1}, V_{1}\right)
$$$$
\cdot\left[\frac{\Phi(T, V)}{\Phi\left(T, V_{1}\right)}-\frac{V}{V_{1}}-1\right.
$$$$
\left.+\frac{\Phi\left(T, V_{1}\right) V}{\Phi(T, V) V_{1}}\right]-\eta_{1} b T_{1}^{*}\left[\frac{\Phi\left(T_{1}, V_{1}\right)}{\Phi\left(T, V_{1}\right)}-1\right.
$$$$
\left.-\ln \left(\frac{\Phi\left(T_{1}, V_{1}\right)}{\Phi\left(T, V_{1}\right)}\right)\right]-\eta_{2} a C_{1}^{*}\left[\frac{\Phi\left(T_{1}, V_{1}\right)}{\Phi\left(T, V_{1}\right)}-1\right.
$$

Using the following equalities,

$$
\begin{aligned}
& \ln \left(\frac{\Phi\left(T\left(t-\tau_{1}\right), V\left(t-\tau_{1}\right)\right)}{\Phi(T, V)}\right) \\
& =\ln \left(\frac{\Phi\left(T\left(t-\tau_{1}\right), V\left(t-\tau_{1}\right)\right) T_{j}^{*}}{\Phi\left(T_{j}, V_{j}\right) T^{*}}\right) \\
& +\ln \left(\frac{\Phi\left(T_{j}, V_{j}\right)}{\Phi\left(T, V_{j}\right)}\right)+\ln \left(\frac{\Phi\left(T, V_{j}\right) V}{\Phi(T, V) V_{j}}\right) \\
& +\ln \left(\frac{V_{j} T^{*}}{V T_{j}^{*}}\right), \\
& \ln \left(\frac{\Phi\left(T\left(t-\tau_{1}\right), V\left(t-\tau_{1}\right)\right)}{\Phi(T, V)}\right) \\
& =\ln \left(\frac{\Phi\left(T\left(t-\tau_{1}\right), V\left(t-\tau_{1}\right)\right) C_{j}^{*}}{\Phi\left(T_{j}, V_{j}\right) C^{*}}\right) \\
& +\ln \left(\frac{\Phi\left(T_{j}, V_{j}\right)}{\Phi\left(T, V_{j}\right)}\right) \ln \left(\frac{\Phi\left(T, V_{j}\right) V}{\Phi(T, V) V_{j}}\right) \\
& +\ln \left(\frac{V_{j} C^{*}}{V C_{j}^{*}}\right), \\
& \ln \left(\frac{T^{*}\left(t-\tau_{2}\right)}{T^{*}}\right) \\
& =\ln \left(\frac{T^{*}\left(t-\tau_{2}\right) V_{j}}{V T_{j}^{*}}\right)+\ln \left(\frac{V T_{j}^{*}}{V_{j} T^{*}}\right), \\
& \ln \left(\frac{C^{*}\left(t-\tau_{3}\right)}{C^{*}}\right) \\
& =\ln \left(\frac{C^{*}\left(t-\tau_{3}\right) V_{j}}{V C_{j}^{*}}\right)+\ln \left(\frac{V C_{j}^{*}}{V_{j} C^{*}}\right),
\end{aligned}
$$$$
\left.-\ln \left(\frac{\Phi\left(T_{1}, V_{1}\right)}{\Phi\left(T, V_{1}\right)}\right)\right]
$$$$
-\eta_{1} b T_{1}^{*}\left[\frac{\Phi\left(T\left(t-\tau_{1}\right), V\left(t-\tau_{1}\right)\right) T_{1}^{*}}{\Phi\left(T_{1}, V_{1}\right) T^{*}}-1\right.
$$$$
\left.-\ln \left(\frac{\Phi\left(T\left(t-\tau_{1}\right), V\left(t-\tau_{1}\right)\right) T_{1}^{*}}{\Phi\left(T_{1}, V_{1}\right) T^{*}}\right)\right]
$$$$
-\eta_{2} a C_{1}^{*}\left[\frac{\Phi\left(T\left(t-\tau_{1}\right), V\left(t-\tau_{1}\right)\right) C_{1}^{*}}{\Phi\left(T_{1}, V_{1}\right) C^{*}}-1\right.
$$$$
\left.-\ln \left(\frac{\Phi\left(T\left(t-\tau_{1}\right), V\left(t-\tau_{1}\right)\right) C_{1}^{*}}{\Phi\left(T_{1}, V_{1}\right) C^{*}}\right)\right]
$$$$
-\eta_{1} b T_{1}^{*}\left[\frac{T^{*}\left(t-\tau_{2}\right) V_{1}}{V T_{1}^{*}}-1\right.
$$$$
\left.-\ln \left(\frac{T^{*}\left(t-\tau_{2}\right) V_{1}}{V T_{1}^{*}}\right)\right]-\eta_{2} a C_{1}^{*}\left[\frac{C^{*}\left(t-\tau_{3}\right) V_{1}}{V C_{1}^{*}}\right.
$$$$
\left.-1-\ln \left(\frac{C^{*}\left(t-\tau_{3}\right) V_{1}}{V C_{1}^{*}}\right)\right]
$$$$
-\eta_{1} b T_{1}^{*}\left[\frac{\Phi\left(T, V_{1}\right) V}{\Phi(T, V) V_{1}}-1-\ln \left(\frac{\Phi\left(T, V_{1}\right) V}{\Phi(T, V) V_{1}}\right)\right]
$$$$
-\eta_{2} a C_{1}^{*}\left[\frac{\Phi\left(T, V_{1}\right) V}{\Phi(T, V) V_{1}}-1-\ln \left(\frac{\Phi\left(T, V_{1}\right) V}{\Phi(T, V) V_{1}}\right)\right]
$$$$
+r \eta_{3}\left(V_{1}-\frac{\mu}{g}\right) Z=d T_{1}\left(1-\frac{\Phi\left(T_{1}, V_{1}\right)}{\Phi\left(T, V_{1}\right)}\right)(1
$$$$
\left.-\frac{T}{T_{1}}\right)+\Phi\left(T_{1}, V_{1}\right)\left(\frac{\Phi(T, V)}{\Phi\left(T, V_{1}\right)}-\frac{V}{V_{1}}\right)(1
$$$$
\left.-\frac{\Phi\left(T, V_{1}\right)}{\Phi(T, V)}\right)-\eta_{1} b T_{1}^{*}\left[H\left(\frac{\Phi\left(T_{1}, V_{1}\right)}{\Phi\left(T, V_{1}\right)}\right)\right.
$$$$
+H\left(\frac{\Phi\left(T\left(t-\tau_{1}\right), V\left(t-\tau_{1}\right)\right) T_{1}^{*}}{\Phi\left(T_{1}, V_{1}\right) T^{*}}\right)
$$$$
\left.+H\left(\frac{T^{*}\left(t-\tau_{2}\right) V_{1}}{V T_{1}^{*}}\right)+H\left(\frac{\Phi\left(T, V_{1}\right) V}{\Phi(T, V) V_{1}}\right)\right]
$$

$j=1,2$, 


$$
\begin{aligned}
& -\eta_{2} a C_{1}^{*}\left[H\left(\frac{\Phi\left(T_{1}, V_{1}\right)}{\Phi\left(T, V_{1}\right)}\right)\right. \\
& +H\left(\frac{\Phi\left(T\left(t-\tau_{1}\right), V\left(t-\tau_{1}\right)\right) C_{1}^{*}}{\Phi\left(T_{1}, V_{1}\right) C^{*}}\right) \\
& \left.+H\left(\frac{C^{*}\left(t-\tau_{3}\right) V_{1}}{V C_{1}^{*}}\right)+H\left(\frac{\Phi\left(T, V_{1}\right) V}{\Phi(T, V) V_{1}}\right)\right] \\
& +r \eta_{3}\left(V_{1}-\frac{\mu}{g}\right) Z .
\end{aligned}
$$

Since $R_{1} \leq 1$, then Conditions C1 and C3 and Lemma 12 imply that $d U_{1} / d t \leq 0$, for all $T, T^{*}, C^{*}, V, Z>0$, where the equality occurs at $S_{1}$. LIP implies that $S_{1}$ is GAS.

Theorem 14. Let $R_{1}>1$ and Conditions C1-C3 be satisfied; then, $S_{2}$ for system (57)-(61) is GAS.

Proof. We consider a Lyapunov functional:

$$
\begin{aligned}
U_{2}= & T-T_{2}-\int_{T_{2}}^{T} \frac{\Phi\left(T_{2}, V_{2}\right)}{\Phi\left(\rho, V_{2}\right)} d \rho+\eta_{1} T_{2}^{*} H\left(\frac{T^{*}}{T_{2}^{*}}\right) \\
& +\eta_{2} C_{2}^{*} H\left(\frac{C^{*}}{C_{2}^{*}}\right)+\eta_{3} V_{2} H\left(\frac{V}{V_{2}}\right) \\
& +\eta_{4} Z_{2} H\left(\frac{Z}{Z_{2}}\right)+\Phi\left(T_{2}, V_{2}\right) \\
& \cdot \int_{0}^{\tau_{1}} H\left(\frac{\Phi(T(t-\theta), V(t-\theta))}{\Phi\left(T_{2}, V_{2}\right)}\right) d \theta \\
& +\eta_{6} T_{2}^{*} \int_{0}^{\tau_{2}} H\left(\frac{T^{*}(t-\theta)}{T_{2}^{*}}\right) d \theta \\
& +\eta_{7} C_{2}^{*} \int_{0}^{\tau_{3}} H\left(\frac{C^{*}(t-\theta)}{C_{2}^{*}}\right) d \theta .
\end{aligned}
$$

Then,

$$
\begin{aligned}
& \frac{d U_{2}}{d t}=\left(1-\frac{\Phi\left(T_{2}, V_{2}\right)}{\Phi\left(T, V_{2}\right)}\right)(\pi-d T-\Phi(T, V)) \\
& +\eta_{1}\left(1-\frac{T_{2}^{*}}{T^{*}}\right) \\
& \cdot\left(e^{-m_{1} \tau_{1}}(1-\xi) \Phi\left(T\left(t-\tau_{1}\right), V\left(t-\tau_{1}\right)\right)\right. \\
& \left.-b T^{*}\right)+\eta_{2}\left(1-\frac{C_{2}^{*}}{C^{*}}\right) \\
& \cdot\left(e^{-m_{1} \tau_{1}} \xi \Phi\left(T\left(t-\tau_{1}\right), V\left(t-\tau_{1}\right)\right)\right. \\
& \left.-a C^{*}\right)+\eta_{3}\left(1-\frac{V_{2}}{V}\right)\left(e^{-m_{2} \tau_{2}} N_{T} b T^{*}\left(t-\tau_{2}\right)\right. \\
& \left.+e^{-m_{3} \tau_{3}} N_{C} a C^{*}\left(t-\tau_{3}\right)-c V-r V Z\right)+\eta_{4}(1
\end{aligned}
$$

$$
\begin{aligned}
& \left.-\frac{Z_{2}}{Z}\right)(g V Z-\mu Z)+\Phi(T, V) \\
& -\Phi\left(T\left(t-\tau_{1}\right), V\left(t-\tau_{1}\right)\right)+\Phi\left(T_{2}, V_{2}\right) \\
& \cdot \ln \left(\frac{\Phi\left(T\left(t-\tau_{1}\right), V\left(t-\tau_{1}\right)\right)}{\Phi(T, V)}\right)+\eta_{6}\left[T^{*}\right. \\
& \left.-T^{*}\left(t-\tau_{2}\right)+T_{2}^{*} \ln \left(\frac{T^{*}\left(t-\tau_{2}\right)}{T^{*}}\right)\right]+\eta_{7}\left[C^{*}\right. \\
& \left.-C^{*}\left(t-\tau_{3}\right)+C_{2}^{*} \ln \left(\frac{C^{*}\left(t-\tau_{3}\right)}{C^{*}}\right)\right] .
\end{aligned}
$$

Using the steady state conditions for $S_{2}$,

$$
\begin{aligned}
\pi & =d T_{2}+\Phi\left(T_{2}, V_{2}\right), \\
(1-\xi) \Phi\left(T_{2}, V_{2}\right) & =e^{m_{1} \tau_{1}} b T_{2}^{*}, \\
\xi \Phi\left(T_{2}, V_{2}\right) & =e^{m_{1} \tau_{1}} a C_{2}^{*}, \\
c V_{2}= & e^{-m_{2} \tau_{2}} N_{T} b T_{2}^{*}+e^{-m_{3} \tau_{3}} N_{C} a C_{2}^{*} \\
& -r V_{2} Z_{2},
\end{aligned}
$$

we get $\Phi\left(T_{2}, V_{2}\right)=\eta_{1} b T_{2}^{*}+\eta_{2} a C_{2}^{*}$, and

$$
\begin{aligned}
\frac{d U_{2}}{d t}= & d T_{2}\left(1-\frac{\Phi\left(T_{2}, V_{2}\right)}{\Phi\left(T, V_{2}\right)}\right)\left(1-\frac{T}{T_{2}}\right) \\
& +\Phi\left(T_{2}, V_{2}\right) \frac{\Phi(T, V)}{\Phi\left(T, V_{2}\right)} \\
& +\eta_{1} b T_{2}^{*}\left(1-\frac{\Phi\left(T_{2}, V_{2}\right)}{\Phi\left(T, V_{2}\right)}\right) \\
& +\eta_{2} a C_{2}^{*}\left(1-\frac{\Phi\left(T_{2}, V_{2}\right)}{\Phi\left(T, V_{2}\right)}\right) \\
& -\eta_{1} b T_{2}^{*} \frac{\Phi\left(T\left(t-\tau_{1}\right), V\left(t-\tau_{1}\right)\right) T_{2}^{*}}{\Phi\left(T_{2}, V_{2}\right) T^{*}} \\
& +\eta_{1} b T_{2}^{*} \\
& -\eta_{2} a C_{2}^{*} \frac{\Phi\left(T\left(t-\tau_{1}\right), V\left(t-\tau_{1}\right)\right) C_{2}^{*}}{\Phi\left(T_{2}, V_{2}\right) C^{*}} \\
& +\eta_{2} a C_{2}^{*}-\eta_{1} b T_{2}^{*} \frac{T^{*}\left(t-\tau_{2}\right) V_{2}}{V T_{2}^{*}} \\
& -\eta_{2} a C_{2}^{*} \frac{C^{*}\left(t-\tau_{3}\right) V_{2}}{V C_{2}^{*}}+\eta_{1} b T_{2}^{*}+\eta_{2} a C_{2}^{*} \\
& -\Phi\left(T_{2}, V_{2}\right) \frac{V}{V_{2}} \\
& +\eta_{1} b T_{2}^{*} \ln \left(\frac{\Phi\left(T\left(t-\tau_{1}\right), V\left(t-\tau_{1}\right)\right)}{\Phi(T, V)}\right)
\end{aligned}
$$




$$
\begin{aligned}
& +\eta_{2} a C_{2}^{*} \ln \left(\frac{\Phi\left(T\left(t-\tau_{1}\right), V\left(t-\tau_{1}\right)\right)}{\Phi(T, V)}\right) \\
& +\eta_{1} b T_{2}^{*} \ln \left(\frac{T^{*}\left(t-\tau_{2}\right)}{T^{*}}\right) \\
& +\eta_{2} a C_{2}^{*} \ln \left(\frac{C^{*}\left(t-\tau_{3}\right)}{C^{*}}\right)
\end{aligned}
$$

Using (97), when $j=2$, we get

$$
\begin{aligned}
& \frac{d U_{2}}{d t}=d T_{2}\left(1-\frac{\Phi\left(T_{2}, V_{2}\right)}{\Phi\left(T, V_{2}\right)}\right)\left(1-\frac{T}{T_{2}}\right) \\
& +\Phi\left(T_{2}, V_{2}\right)\left[\frac{\Phi(T, V)}{\Phi\left(T, V_{2}\right)}-\frac{V}{V_{2}}-1+\frac{\Phi\left(T, V_{2}\right) V}{\Phi(T, V) V_{2}}\right] \\
& -\eta_{1} b T_{2}^{*}\left[\frac{\Phi\left(T_{2}, V_{2}\right)}{\Phi\left(T, V_{2}\right)}-1-\ln \left(\frac{\Phi\left(T_{2}, V_{2}\right)}{\Phi\left(T, V_{2}\right)}\right)\right] \\
& -\eta_{2} a C_{2}^{*}\left[\frac{\Phi\left(T_{2}, V_{2}\right)}{\Phi\left(T, V_{2}\right)}-1-\ln \left(\frac{\Phi\left(T_{2}, V_{2}\right)}{\Phi\left(T, V_{2}\right)}\right)\right] \\
& -\eta_{1} b T_{2}^{*}\left[\frac{\Phi\left(T\left(t-\tau_{1}\right), V\left(t-\tau_{1}\right)\right) T_{2}^{*}}{\Phi\left(T_{2}, V_{2}\right) T^{*}}-1\right. \\
& \left.-\ln \left(\frac{\Phi\left(T\left(t-\tau_{1}\right), V\left(t-\tau_{1}\right)\right) T_{2}^{*}}{\Phi\left(T_{2}, V_{2}\right) T^{*}}\right)\right] \\
& -\eta_{2} a C_{2}^{*}\left[\frac{\Phi\left(T\left(t-\tau_{1}\right), V\left(t-\tau_{1}\right)\right) C_{2}^{*}}{\Phi\left(T_{2}, V_{2}\right) C^{*}}-1\right. \\
& \left.-\ln \left(\frac{\Phi\left(T\left(t-\tau_{1}\right), V\left(t-\tau_{1}\right)\right) C_{2}^{*}}{\Phi\left(T_{2}, V_{2}\right) C^{*}}\right)\right] \\
& -\eta_{1} b T_{2}^{*}\left[\frac{T^{*}\left(t-\tau_{2}\right) V_{2}}{V T_{2}^{*}}-1\right. \\
& \left.-\ln \left(\frac{T^{*}\left(t-\tau_{2}\right) V_{2}}{V T_{2}^{*}}\right)\right]-\eta_{2} a C_{2}^{*}\left[\frac{C^{*}\left(t-\tau_{3}\right) V_{2}}{V C_{2}^{*}}\right. \\
& \left.-1-\ln \left(\frac{C^{*}\left(t-\tau_{3}\right) V_{2}}{V C_{2}^{*}}\right)\right] \\
& -\eta_{1} b T_{2}^{*}\left[\frac{\Phi\left(T, V_{2}\right) V}{\Phi(T, V) V_{2}}-1-\ln \left(\frac{\Phi\left(T, V_{2}\right) V}{\Phi(T, V) V_{2}}\right)\right] \\
& -\eta_{2} a C_{2}^{*}\left[\frac{\Phi\left(T, V_{2}\right) V}{\Phi(T, V) V_{2}}-1-\ln \left(\frac{\Phi\left(T, V_{2}\right) V}{\Phi(T, V) V_{2}}\right)\right] \\
& =d T_{2}\left(1-\frac{\Phi\left(T_{2}, V_{2}\right)}{\Phi\left(T, V_{2}\right)}\right)\left(1-\frac{T}{T_{2}}\right)+\Phi\left(T_{2}, V_{2}\right) \\
& \cdot\left(\frac{\Phi(T, V)}{\Phi\left(T, V_{2}\right)}-\frac{V}{V_{2}}\right)\left(1-\frac{\Phi\left(T, V_{2}\right)}{\Phi(T, V)}\right)
\end{aligned}
$$

$$
\begin{aligned}
& -\eta_{1} b T_{2}^{*}\left[H\left(\frac{\Phi\left(T_{2}, V_{2}\right)}{\Phi\left(T, V_{2}\right)}\right)\right. \\
& +H\left(\frac{\Phi\left(T\left(t-\tau_{1}\right), V\left(t-\tau_{1}\right)\right) T_{2}^{*}}{\Phi\left(T_{2}, V_{2}\right) T^{*}}\right) \\
& \left.+H\left(\frac{T^{*}\left(t-\tau_{2}\right) V_{2}}{V T_{2}^{*}}\right)+H\left(\frac{\Phi\left(T, V_{2}\right) V}{\Phi(T, V) V_{2}}\right)\right] \\
& -\eta_{2} a C_{2}^{*}\left[H\left(\frac{\Phi\left(T_{1}, V_{1}\right)}{\Phi\left(T, V_{1}\right)}\right)\right. \\
& +H\left(\frac{\Phi\left(T\left(t-\tau_{1}\right), V\left(t-\tau_{1}\right)\right) C_{2}^{*}}{\Phi\left(T_{2}, V_{2}\right) C^{*}}\right) \\
& \left.+H\left(\frac{C^{*}\left(t-\tau_{3}\right) V_{2}}{V C_{2}^{*}}\right)+H\left(\frac{\Phi\left(T, V_{2}\right) V}{\Phi(T, V) V_{2}}\right)\right] .
\end{aligned}
$$

It is easy to show that $S_{2}$ is GAS.

\section{Numerical Simulations}

In this section, we show an example of the general model (50)(57) where Conditions C1-C3 can be satisfied:

$$
\begin{aligned}
\dot{T}(t)= & \pi-d T(t)-\frac{(1-\varepsilon) k T(t) V(t)}{1+\omega T(t)+\beta V(t)} \\
\dot{T}^{*}(t)= & e^{-m_{1} \tau_{1}}(1-\xi) \frac{(1-\varepsilon) k T\left(t-\tau_{1}\right) V\left(t-\tau_{1}\right)}{1+\omega T\left(t-\tau_{1}\right)+\beta V\left(t-\tau_{1}\right)} \\
& -b T^{*}(t), \\
\dot{C}^{*}(t)= & e^{-m_{1} \tau_{1}} \xi \frac{(1-\varepsilon) k T\left(t-\tau_{1}\right) V\left(t-\tau_{1}\right)}{1+\omega T\left(t-\tau_{1}\right)+\beta V\left(t-\tau_{1}\right)} \\
& -a C^{*}(t), \\
\dot{V}(t)= & e^{-m_{2} \tau_{2}} N_{T} b T^{*}\left(t-\tau_{2}\right) \\
& +e^{-m_{3} \tau_{3}} N_{C} a C^{*}\left(t-\tau_{3}\right)-r V(t) Z(t) \\
& -c V(t), \\
\dot{Z}(t)= & g V(t) Z(t)-\mu Z(t),
\end{aligned}
$$

where $\omega, \beta \geqslant 0$. In this example, the incidence rate is given by

$$
\Phi(T, V)=\frac{(1-\varepsilon) k T V}{1+\omega T+\beta V} .
$$

We have the following:

$$
\begin{aligned}
& \Phi(T, V)>0, \\
& \Phi(T, 0)=\Phi(0, V)=0,
\end{aligned}
$$




$$
\begin{aligned}
& \frac{\partial \Phi(T, V)}{\partial T}=\frac{(1-\varepsilon) k V(1+\beta V)}{(1+\omega T+\beta V)^{2}}>0, \\
& \forall T>0, V>0, \\
& \frac{\partial \Phi(T, V)}{\partial V}=\frac{(1-\varepsilon) k T(1+\omega T)}{(1+\omega T+\beta V)^{2}}>0, \\
& \forall T>0, V>0, \\
& \frac{\partial \Phi(T, 0)}{\partial V}=\frac{(1-\varepsilon) k T}{1+\omega T}>0, \quad \forall T>0, \\
& \Phi(T, V)=\frac{(1-\varepsilon) k T V}{1+\omega T+\beta V} \leqslant \frac{(1-\varepsilon) k T V}{1+\omega T} \\
& =V \frac{\partial \Phi(T, 0)}{\partial V}, \quad \forall T>0, V>0, \\
& \left(\frac{\Phi(T, V)}{\Phi\left(T, V_{i}\right)}-\frac{V}{V_{i}}\right)\left(1-\frac{\Phi\left(T, V_{i}\right)}{\Phi(T, V)}\right) \\
& =\frac{-\beta(1+\omega T)\left(V-V_{i}\right)^{2}}{V_{i}\left(1+\omega T+\beta V_{i}\right)(1+\omega T+\beta V)} \leqslant 0, \\
& \forall T, V>0, i=1,2 .
\end{aligned}
$$

Then, Conditions $\mathrm{C} 1-\mathrm{C} 3$ are satisfied. The parameters $R_{0}$ and $R_{1}$ will be

$$
\begin{aligned}
& R_{0} \\
& =\frac{(1-\varepsilon) k T_{0}\left[e^{-\left(m_{1} \tau_{1}+m_{2} \tau_{2}\right)}(1-\xi) N_{T}+e^{-\left(m_{1} \tau_{1}+m_{3} \tau_{3}\right)} \xi N_{C}\right]}{c\left(1+\omega T_{0}\right)}, \\
& R_{1} \\
& =\frac{(1-\varepsilon) k T_{2}\left[e^{-\left(m_{1} \tau_{1}+m_{2} \tau_{2}\right)}(1-\xi) N_{T}+e^{-\left(m_{1} \tau_{1}+m_{3} \tau_{3}\right)} \xi N_{C}\right]}{c\left(1+\omega T_{2}+\beta V_{2}\right)},
\end{aligned}
$$

where

$$
\begin{aligned}
& T_{2}=\frac{1}{2 \omega}\left(\omega T_{0}-\left(1+\zeta V_{2}\right)\right. \\
& \left.+\sqrt{\left[\left(1+\zeta V_{2}\right)-\omega T_{0}\right]^{2}+4 \omega T_{0}\left(1+\beta V_{2}\right)}\right), \\
& \quad V_{2}=\frac{\mu}{g}, \zeta=\beta+\frac{(1-\varepsilon) k}{d} .
\end{aligned}
$$

The values of some parameters of model (104) are listed in Table 1 . The remaining parameters of the model will be chosen varied. Without loss of generality, we let $\tau_{e}=\tau_{1}=\tau_{2}=$ $\tau_{3}$. We have used MATLAB for the numerical computations. Now, we study the following cases.
TABLE 1: The parameters of model (104).

\begin{tabular}{lc}
\hline Parameter & Value $^{-3}$ dalls mm $^{-1}$ day $^{-1}$ \\
$d$ & 0.01 day $^{-1}$ \\
$b$ & 0.3 day $^{-1}$ \\
$\omega$ & 0.05 cells $^{-1} \mathrm{~mm}^{3}$ \\
$\beta$ & 0.05 virus $^{-1} \mathrm{~mm}^{3}$ \\
$a$ & 0.25 day $^{-1}$ \\
$N_{T}$ & 100 virus cells $^{-1}$ \\
$\xi$ & 0.5 \\
$N_{C}$ & 50 virus cells $^{-1}$ \\
$r$ & 0.01 cells $^{-1} \mathrm{~mm}^{3}$ day $^{-1}$ \\
$\mu$ & 0.05 day $^{-1}$ \\
$m_{1}$ & 1 day $^{-1}$ \\
$m_{2}$ & 1 day $^{-1}$ \\
$m_{3}$ & 1 day $^{-1}$ \\
$c$ & 3 day $^{-1}$ \\
\hline
\end{tabular}

Case 1 (effect of the parameters $k$ and $g$ on the stability of the system). Now, we confirm the results of Theorems 11-14. The evolution of the dynamics of model (104) was observed over a time interval $[0,1000]$. We have chosen the initial conditions as follows: $T(0)=600, T^{*}(0)=10, C^{*}(0)=5, V(0)=10$, and $Z(0)=80$. We use three sets for the parameters $k$ and $g$ to get the following three subcases. We fix the values $\tau_{e}=0.5$ and $\varepsilon=0$ :

(i) $R_{0} \leq 1$. The values of $k$ and $g$ are chosen as $k=0.002$ virus $^{-1} \mathrm{~mm}^{3}$ day $^{-1}$ and $g=0.0005$ virus $^{-1} \mathrm{~mm}^{3}$ day $^{-1}$. The values of the two bifurcation parameters are computed as $R_{0}=0.3607<1$ and $R_{1}=0.3114<1$. This means that $S_{0}$ is GAS. One can see from Figures 1-5 that the numerical results confirm the results of Theorem 11 . We observe that the states of the system eventually approach the steady state $S_{0}(1000,0,0,0,0)$. In this case, the HIV will be removed from the blood.

(ii) $R_{1} \leq 1<R_{0}$. We take the values $k=$ 0.01 virus $^{-1} \mathrm{~mm}^{3}$ day $^{-1}$ and $g=0.0005$ virus ${ }^{-1} \mathrm{~mm}^{3}$ day $^{-1}$. In this case, $R_{1}=0.8291<$ $1<R_{0}=1.8033$. According to Theorem 13, $S_{1}$ is GAS. From Figures $1-5$, we can see that there is a consistency between the numerical and theoretical results of Theorem 13. Moreover, the states of the system converge to the steady $S_{1}(120.22,8.89,10.67,80.91,0)$.

(iii) $R_{1}>1$. We choose $k=0.01$ virus $^{-1} \mathrm{~mm}^{3}$ day $^{-1}$ and $g=0.002$ virus $^{-1} \mathrm{~mm}^{3} \mathrm{day}^{-1}$. Then, we compute $R_{0}=1.8033>1$ and $R_{1}=1.6976>1$. From Figures $1-5$, we can see that the states of the system approach the infected steady state with humoral immune response $S_{2}(538.56,4.66,5.60,25.00,209.27)$. This 


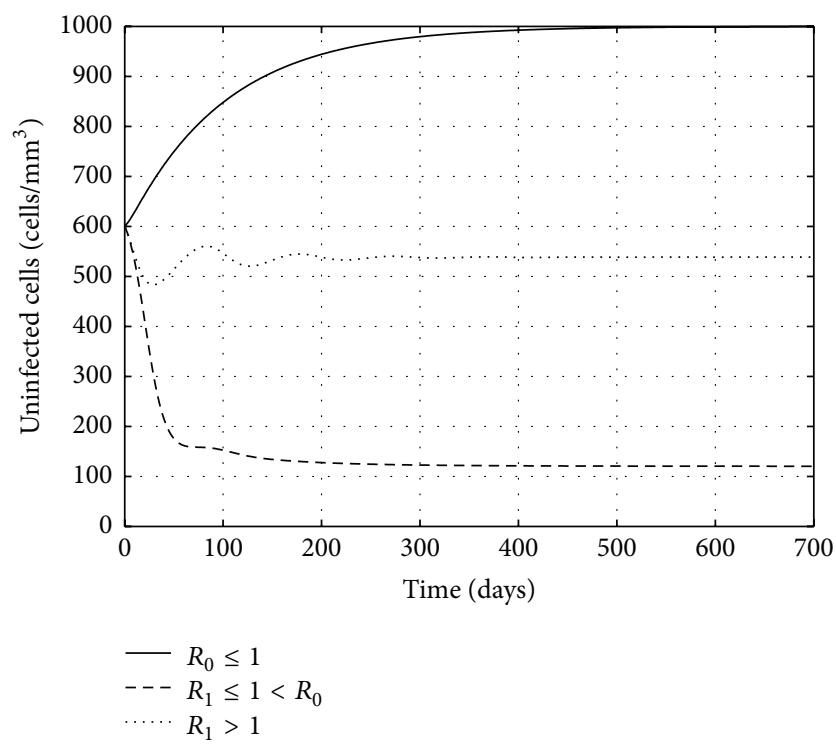

FIgURE 1: The concentration of uninfected $\mathrm{CD} 4^{+} \mathrm{T}$ cells for model (104).

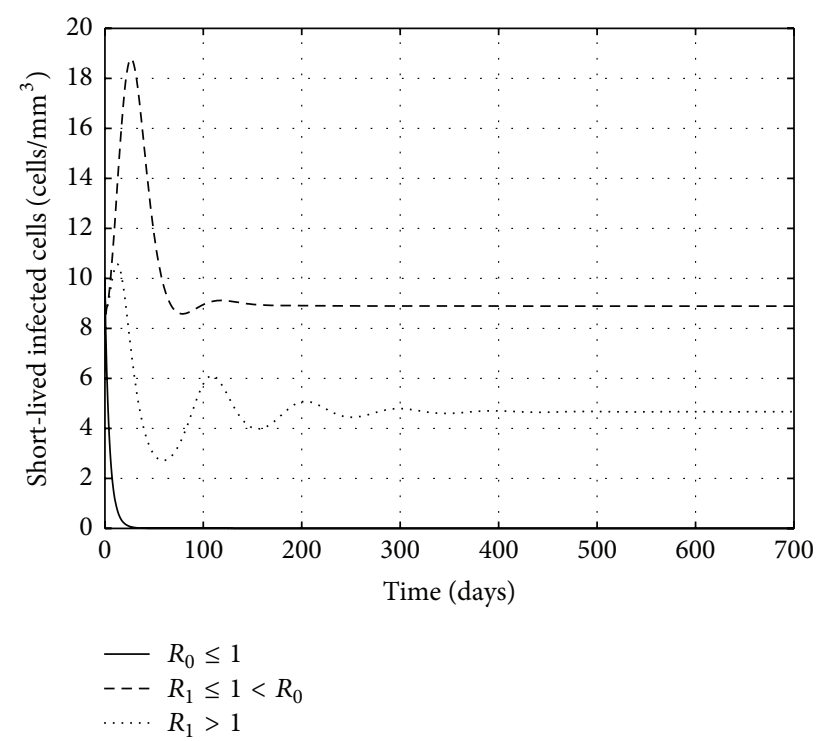

FIGURE 2: The concentration of short-lived infected cells for model (104).

supports the results of Theorem 14 that the infected steady state without humoral immune response $S_{2}$ is GAS.

Case 2 (effect of the parameter $\varepsilon$ on the stability of the system). For this case, we take $\tau_{e}=0.5 \mathrm{day}^{-1}, k=0.01$ virus ${ }^{-1} \mathrm{~mm}^{3} \mathrm{day}^{-1}$, and $g=0.001$ virus $^{-1} \mathrm{~mm}^{3} \mathrm{day}^{-1}$. In Figures 6-10, we show the effect of the drug efficacy $\varepsilon$ on the stability of the steady states of the system. We can see from Figures 6-10 that as the drug efficacy $\varepsilon$ is increased, the concentration of uninfected $\mathrm{CD} 4^{+} \mathrm{T}$ cells is increased,

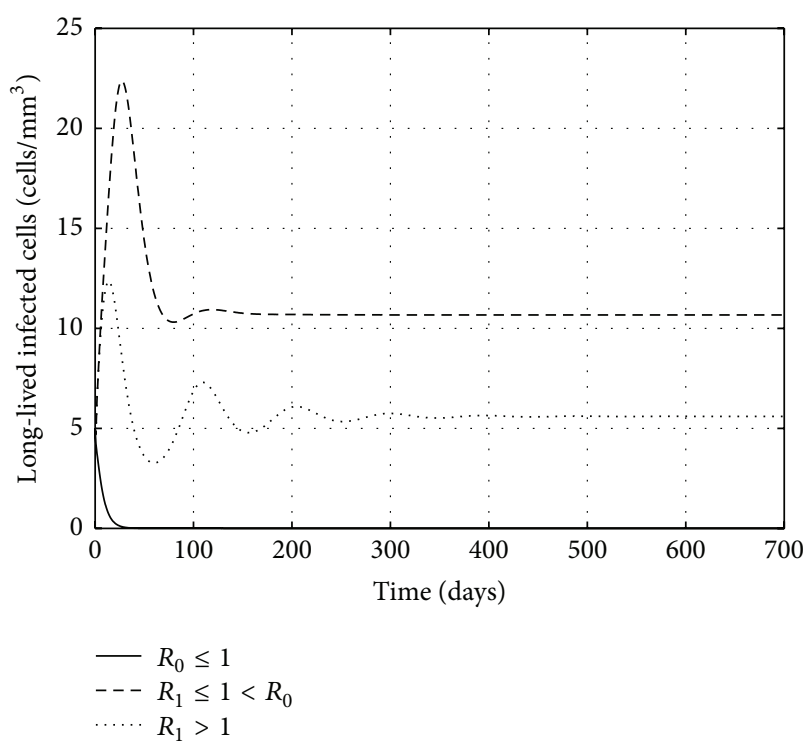

FIGURE 3: The concentration of long-lived chronically infected cells for model (104).

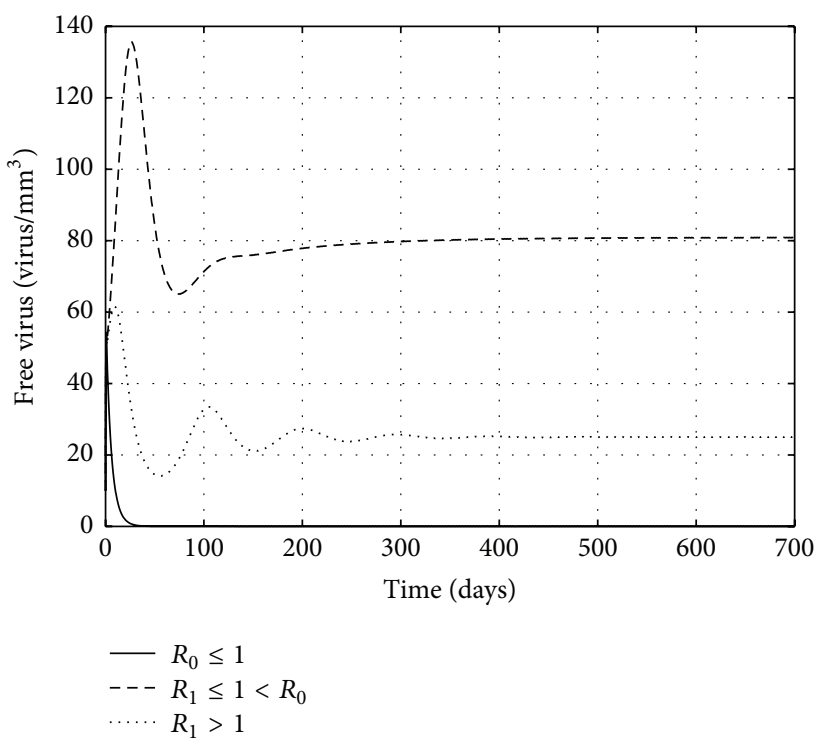

FIgURE 4: The concentration of free virus particles for model (104).

while the concentrations of free virus particles, B cells, shortlived infected cells, and long-lived chronically infected cells are decreased. From these figures and Table 2, we can see that the values of $R_{0}$ and $R_{1}$ are decreased as $\varepsilon$ is increased. Using the values of the parameters given in Table 1, we obtain the following:

(i) If $0 \leq \varepsilon<0.3730$, then $S_{2}$ exists and it is GAS.

(ii) If $0.3730 \leq \varepsilon<0.4455$, then $S_{1}$ exists and it is GAS.

(iii) If $0.4455 \leq \varepsilon<1$, then $S_{0}$ is GAS.

It means that the numerical results and the results of Theorems 11-14 are compatible. In this case, the treatment 


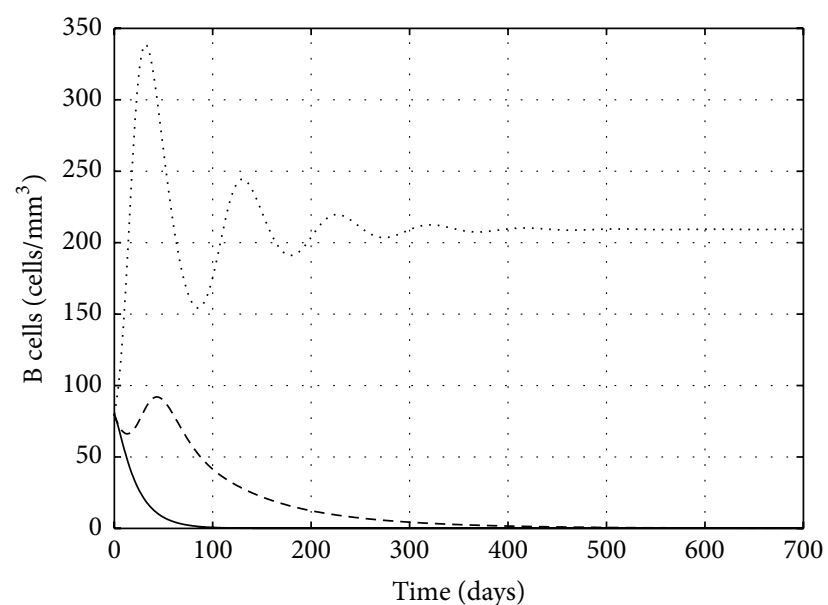

$$
\begin{array}{ll}
- & R_{0} \leq 1 \\
--- & R_{1} \leq 1<R_{0} \\
\cdots \cdots & R_{1}>1
\end{array}
$$

FIGURE 5: The concentration of B cells for model (104).

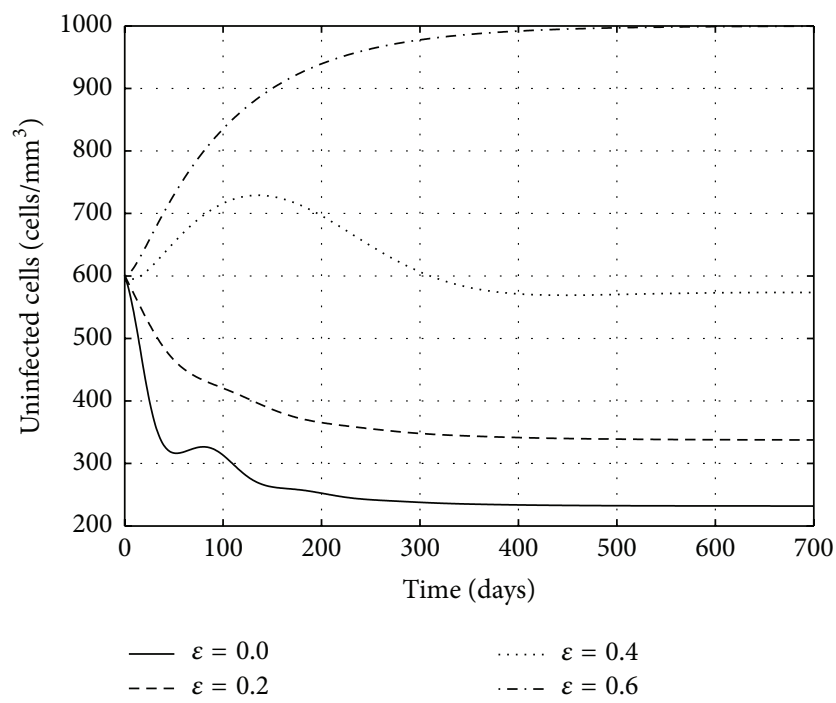

FIGURE 6: The concentration of uninfected $\mathrm{CD} 4^{+} \mathrm{T}$ cells for model (104).

TABLE 2: The values of equilibria, $R_{0}$, and $R_{1}$ for model (104) with different values of $\varepsilon$.

\begin{tabular}{lccc}
\hline & Equilibria & $R_{0}$ & $R_{1}$ \\
\hline$\varepsilon=0.0$ & $E_{2}(231.88,7.77,9.32,50,123.86)$ & 1.8033 & 1.4129 \\
$\varepsilon=0.2$ & $E_{2}(337.44,6.70,8.04,50,65.61)$ & 1.4427 & 1.2187 \\
$\varepsilon=0.3730$ & $E_{1}(456.34,5.50,6.60,50,0)$ & 1.1308 & 1 \\
$\varepsilon=0.4$ & $E_{1}(572.42,4.32,5.19,39.32,0)$ & 1.0820 & 0.9624 \\
$\varepsilon=0.4455$ & $E_{0}(1000,0,0,0,0)$ & 1 & 0.8974 \\
$\varepsilon=0.6$ & $E_{0}(1000,0,0,0,0)$ & 0.7213 & 0.6632 \\
$\varepsilon=0.8$ & $E_{0}(1000,0,0,0,0)$ & 0.3607 & 0.3388 \\
\hline
\end{tabular}

with sufficient drug efficacy can succeed to clear the HIV from the plasma.

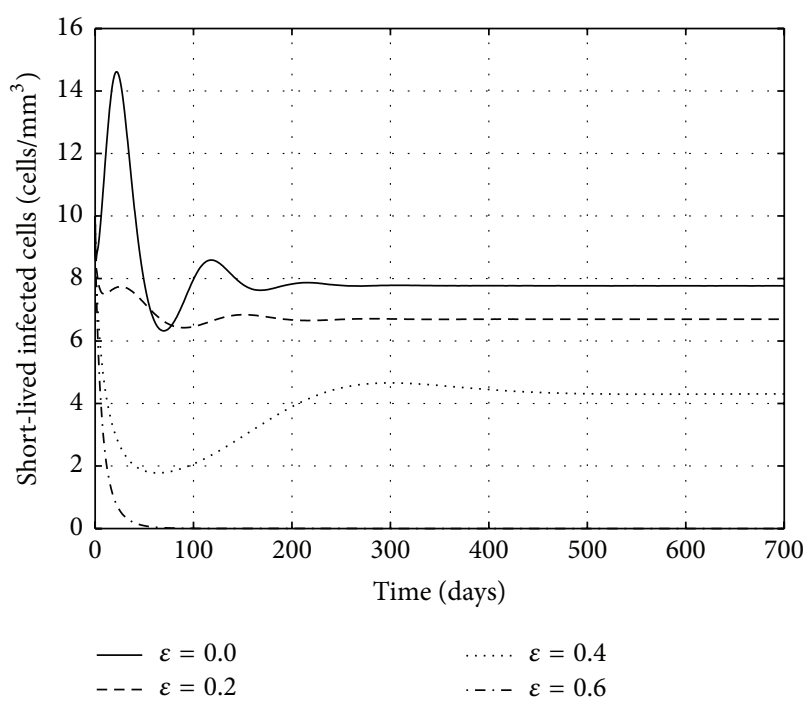

FIGURE 7: The concentration of short-lived infected cells for model (104).

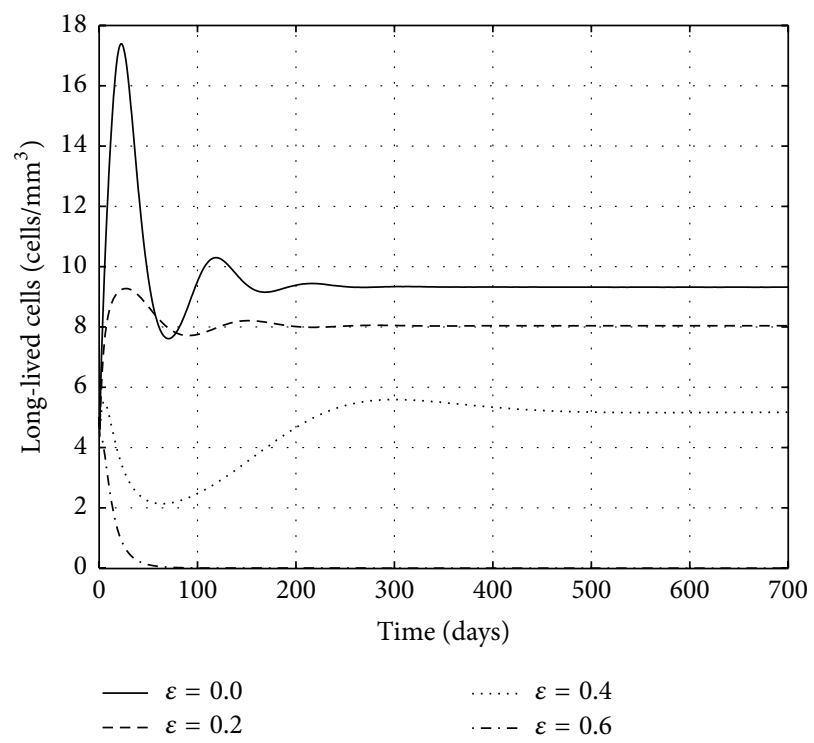

FIGURE 8: The concentration of long-lived chronically infected cells for model (104).

Case 3 (effect of the parameter $\tau_{e}$ on the stability of the system). We fix the parameters $\varepsilon=0.4, k=0.01$ virus ${ }^{-1} \mathrm{~mm}^{3}$ day $^{-1}$, and $g=0.001$ virus $^{-1} \mathrm{~mm} \mathrm{~m}^{3}$ day $^{-1}$. In Figures 11-15, we show the effect of the time delay parameter $\tau_{e}$ on the stability of the steady states of the system. We can see from Figures 11-15 that the time delay parameter $\tau_{e}$ plays a similar role as the drug efficacy parameter $\varepsilon$. From these figures and Table 3 , we can see that the values of $R_{0}$ and $R_{1}$ are decreased when $\tau_{e}$ is increased. Using the values of the parameters given in Table 1, we obtain the following:

(i) If $0 \leq \tau_{e}<0.4808$, then $S_{2}$ exists and it is GAS. 


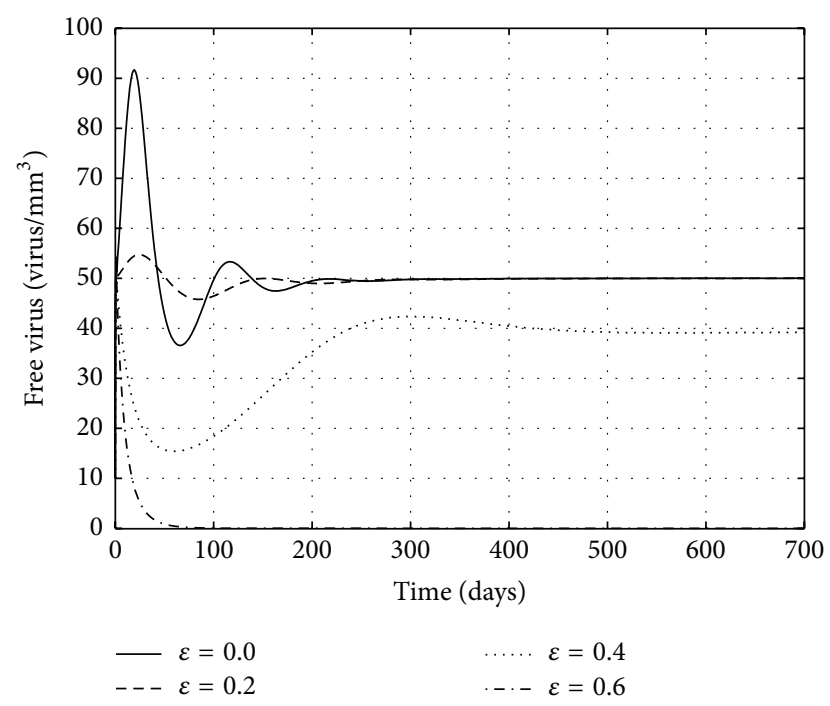

FIGURE 9: The concentration of free virus particles for model (104).

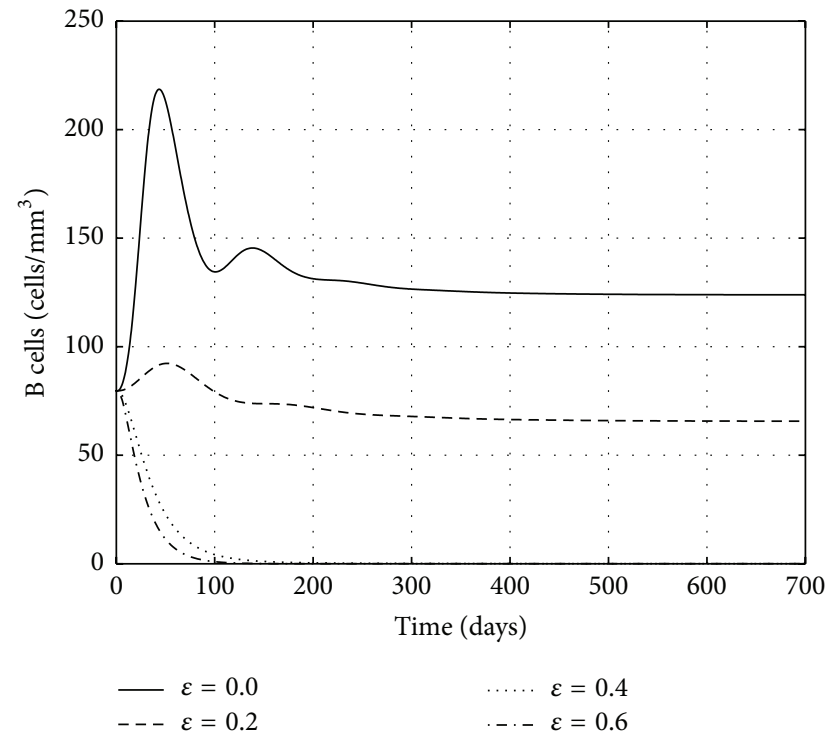

FIGURE 10: The concentration of B cells for model (104).

TABLE 3: The values of equilibria, $R_{0}$, and $R_{1}$ for model (104) with different values of $\tau_{e}$.

\begin{tabular}{lccc}
\hline Delay & Equilibria & $R_{0}$ & $R_{1}$ \\
\hline$\tau_{e}=0.0$ & $E_{2}(476.81,8.72,10.46,50 ., 484.77)$ & 2.9412 & 2.6160 \\
$\tau_{e}=0.1$ & $E_{2}(476.81,7.89,9.47,50,342.53)$ & 2.4080 & 2.1418 \\
$\tau_{e}=0.3$ & $E_{2}(476.81,6.46,7.75,50,130.70)$ & 1.6142 & 1.4357 \\
$\tau_{e}=0.4808$ & $E_{1}(476.81,5.39,6.47,50,0)$ & 1.1243 & 1.0 \\
$\tau_{e}=0.5$ & $E_{1}(572.42,4.32,5.18,39.32,0)$ & 1.0820 & 0.9624 \\
$\tau_{e}=0.5394$ & $E_{0}(1000,0,0,0,0)$ & 1.0 & 0.8894 \\
$\tau_{e}=0.7$ & $E_{0}(1000,0,0,0,0)$ & 0.7253 & 0.6451 \\
$\tau_{e}=1.0$ & $E_{0}(1000,0,0,0,0)$ & 0.3981 & 0.3540 \\
$\tau_{e}=2.0$ & $E_{0}(1000,0,0,0,0)$ & 0.0539 & 0.0479 \\
\hline
\end{tabular}

(ii) If $0.4808 \leq \tau_{e}<0.5394$, then $S_{1}$ exists and it is GAS.

(iii) If $0.5394 \leq \tau_{e}$, then $S_{0}$ is GAS.

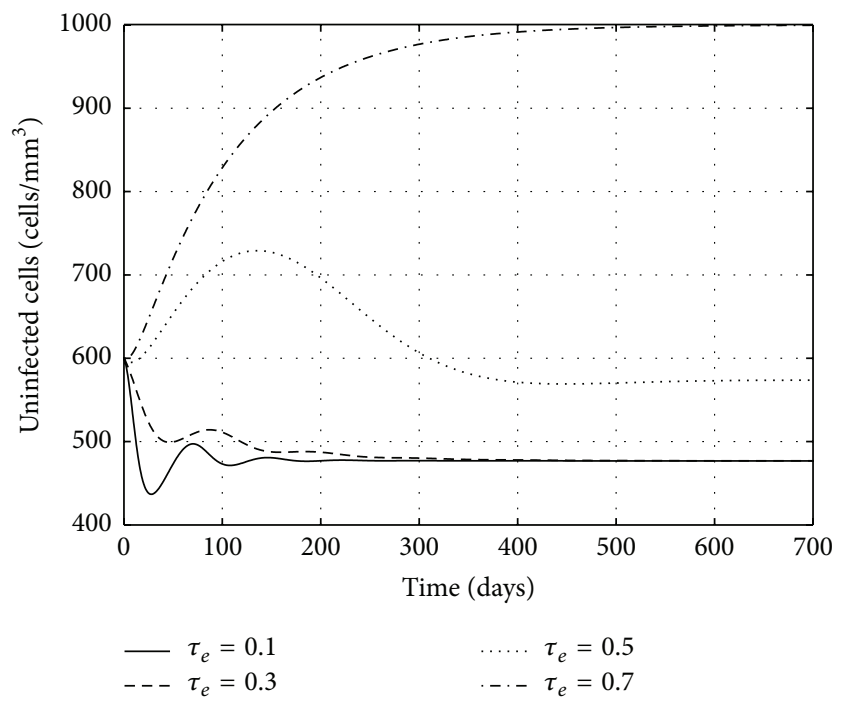

FIGURE 11: The concentration of uninfected $\mathrm{CD} 4^{+} \mathrm{T}$ cells for model (104).

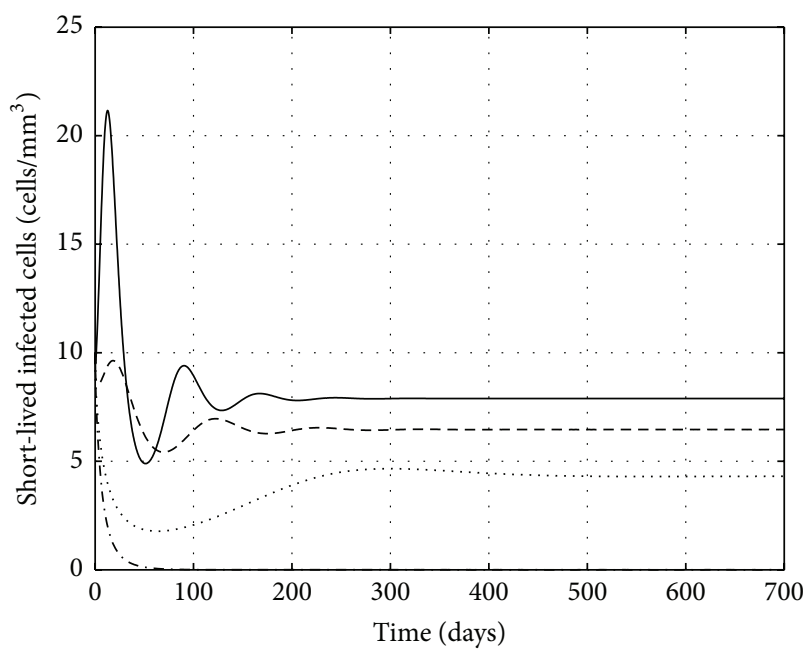

$$
\begin{aligned}
& -\tau_{e}=0.1 \quad \cdots \cdots \tau_{e}=0.5 \\
& --\tau_{e}=0.3 \quad \cdots-\tau_{e}=0.7
\end{aligned}
$$

FIGURE 12: The concentration of short-lived infected cells for model (104).

Figures 11-15 show that the numerical results are also compatible with the results of Theorems 11-14.

\section{Conclusions}

In this paper, we have proposed three HIV infection models with humoral immune response and two types of infected cells. The models incorporate two types of discrete delays. The incidence rate of infection is given by bilinear, saturated functional response and general nonlinear function in the 


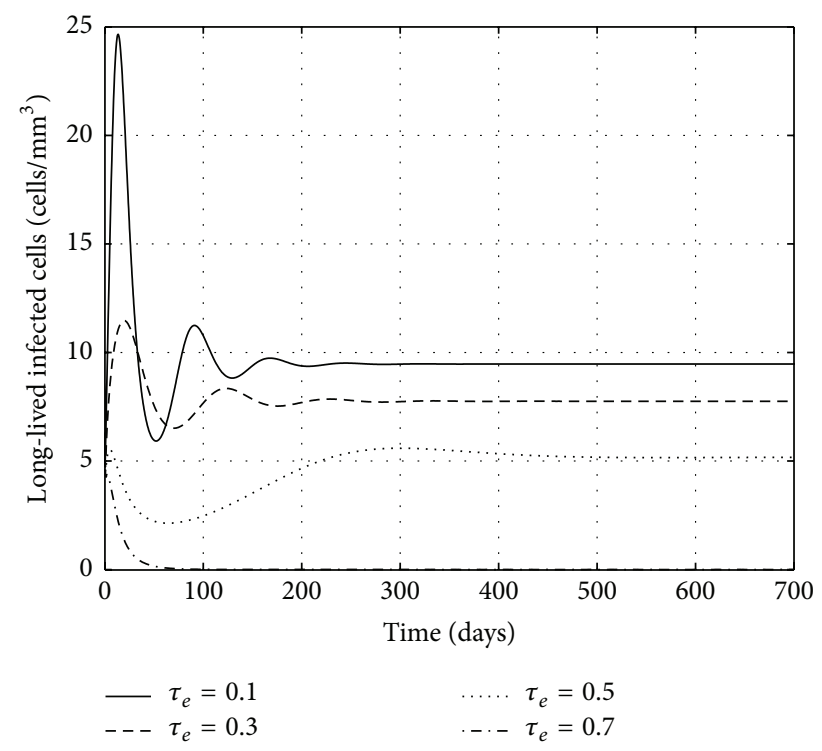

FIGURE 13: The concentration of long-lived chronically infected cells for model (104).

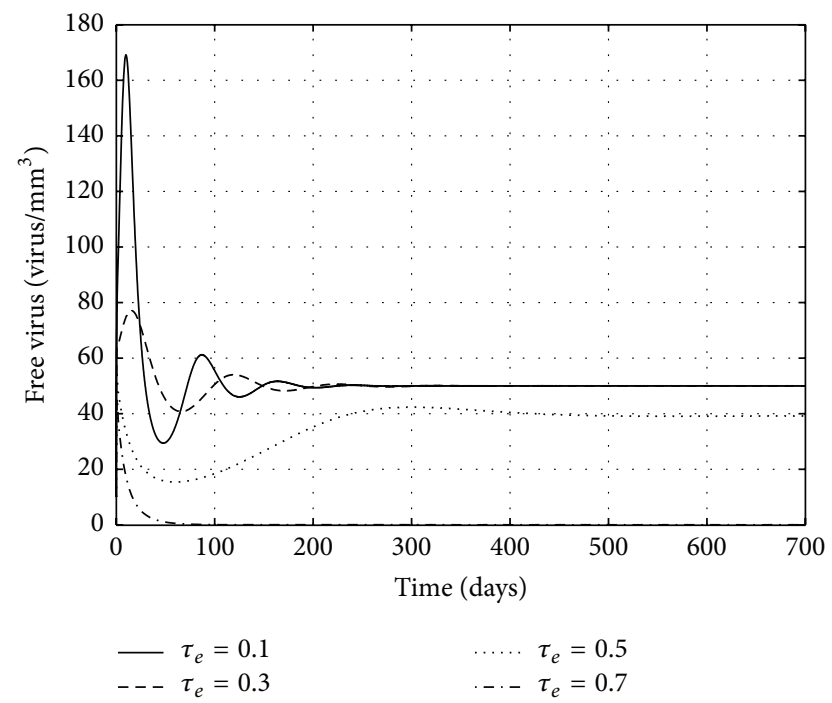

FIGURE 14: The concentration of free virus particles for model (104).

first, second, and third model, respectively. We have derived two bifurcation parameters, $R_{0}$ and $R_{1}$. The global stability of all steady states of the models has been established using Lyapunov method. We have presented an example for the general incidence rate and performed numerical simulations to support our theoretical results.

\section{Conflict of Interests}

The authors declare that there is no conflict of interests regarding the publication of this paper.

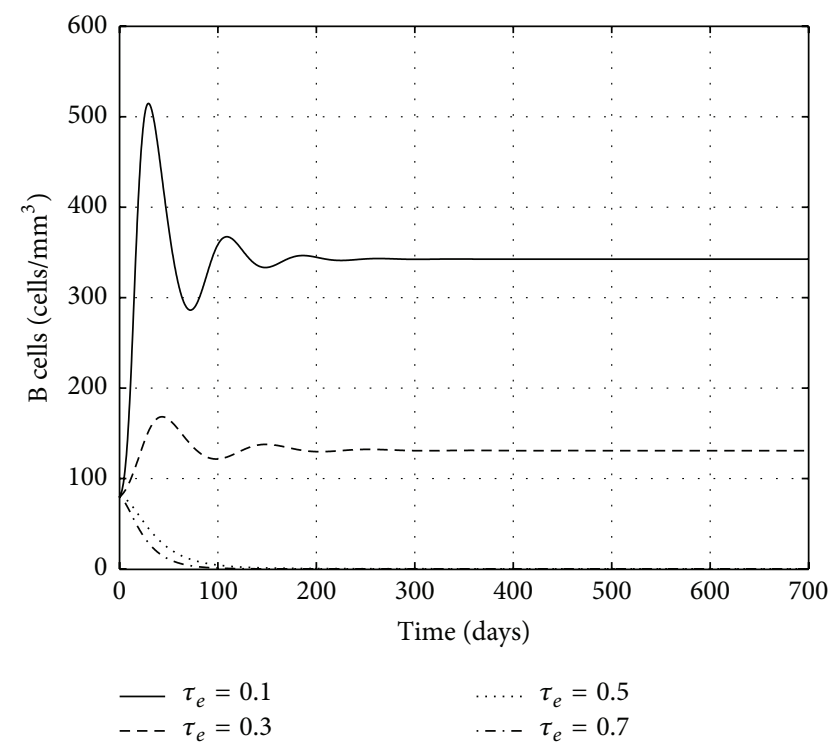

FIgURE 15: The concentration of B cells for model (104).

\section{Acknowledgments}

This paper was funded by the Deanship of Scientific Research (DSR), King Abdulaziz University, Jeddah. The authors, therefore, acknowledge with thanks DSR technical and financial support. The authors are also grateful to Professor Zizhen Zhang for constructive suggestions and valuable comments, which improve the quality of the paper.

\section{References}

[1] M. A. Nowak and C. R. M. Bangham, "Population dynamics of immune responses to persistent viruses," Science, vol. 272, no. 5258, pp. 74-79, 1996.

[2] P. W. Nelson, J. D. Murray, and A. S. Perelson, "A model of HIV-1 pathogenesis that includes an intracellular delay," Mathematical Biosciences, vol. 163, no. 2, pp. 201-215, 2000.

[3] M. A. Nowak and R. M. May, Virus Dynamics: Mathematical Principles of Immunology and Virology, Oxford University Press, Oxford, UK, 2000.

[4] D. S. Callaway and A. S. Perelson, "HIV-1 infection and low steady state viral loads," Bulletin of Mathematical Biology, vol. 64, no. 1, pp. 29-64, 2002.

[5] A. M. Elaiw and S. A. Azoz, "Global properties of a class of HIV infection models with Beddington-DeAngelis functional response," Mathematical Methods in the Applied Sciences, vol. 36, pp. 383-394, 2013.

[6] A. M. Elaiw, "Global properties of a class of HIV models," Nonlinear Analysis. Real World Applications, vol. 11, no. 4, pp. 2253-2263, 2010.

[7] A. M. Elaiw, "Global properties of a class of virus infection models with multitarget cells," Nonlinear Dynamics, vol. 69, no. 1-2, pp. 423-435, 2012.

[8] A. M. Elaiw and A. S. Alsheri, "Global dynamics of HIV infection of $\mathrm{CD}^{+} \mathrm{T}$ cells and macrophages," Discrete Dynamics in Nature and Society, vol. 2013, Article ID 264759, 8 pages, 2013. 
[9] P. W. Nelson and A. S. Perelson, "Mathematical analysis of delay differential equation models of HIV-1 infection," Mathematical Biosciences, vol. 179, no. 1, pp. 73-94, 2002.

[10] Y. Wang, L. Liu, X. Zhang, and Y. Wu, "Positive solutions of an abstract fractional semipositone differential system model for bioprocesses of HIV infection," Applied Mathematics and Computation, vol. 258, pp. 312-324, 2015.

[11] P. Yu, J. Huang, and J. Jiang, "Dynamics of an HIV-1 infection model with cell mediated immunity," Communications in Nonlinear Science and Numerical Simulation, vol. 19, no. 10, pp. 3827-3844, 2014.

[12] X. Chen, L. Huang, and P. Yu, "Dynamic behaviors of a class of HIV compartmental models," Communications in Nonlinear Science and Numerical Simulation, vol. 23, no. 1-3, pp. 115-128, 2015.

[13] X. Y. Zhou, X. Y. Shi, Z. H. Zhang, and X. Y. Song, "Dynamical behavior of a virus dynamics model with CTL immune response," Applied Mathematics and Computation, vol. 213, no. 2, pp. 329-347, 2009.

[14] A. Murase, T. Sasaki, and T. Kajiwara, "Stability analysis of pathogen-immune interaction dynamics," Journal of Mathematical Biology, vol. 51, no. 3, pp. 247-267, 2005.

[15] M. A. Obaid and A. M. Elaiw, "Stability of virus infection models with antibodies and chronically infected cells," Abstract and Applied Analysis, vol. 2014, Article ID 650371, 12 pages, 2014.

[16] T. Wang, Z. Hu, and F. Liao, "Stability and Hopf bifurcation for a virus infection model with delayed humoral immunity response," Journal of Mathematical Analysis and Applications, vol. 411, no. 1, pp. 63-74, 2014.

[17] N. M. Dixit and A. S. Perelson, "Complex patterns of viral load decay under antiretroviral therapy: influence of pharmacokinetics and intracellular delay," Journal of Theoretical Biology, vol. 226, no. 1, pp. 95-109, 2004.

[18] M. Y. Li and H. Shu, "Global dynamics of an in-host viral model with intracellular delay," Bulletin of Mathematical Biology, vol. 72, no. 6, pp. 1492-1505, 2010.

[19] M. Y. Li and H. Shu, "Impact of intracellular delays and targetcell dynamics on in vivo viral infections," SIAM Journal on Applied Mathematics, vol. 70, no. 7, pp. 2434-2448, 2010.

[20] D. Li and W. Ma, "Asymptotic properties of a HIV-1 infection model with time delay," Journal of Mathematical Analysis and Applications, vol. 335, no. 1, pp. 683-691, 2007.

[21] Z. Yuan and X. Zou, "Global threshold dynamics in an HIV virus model with nonlinear infection rate and distributed invasion and production delays," Mathematical Biosciences and Engineering, vol. 10, no. 2, pp. 483-498, 2013.

[22] J. K. Hale and S. V. Lunel, Introduction To Functional Differential Equations, Springer, New York, NY, USA, 1993. 


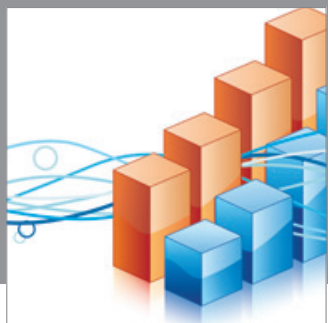

Advances in

Operations Research

mansans

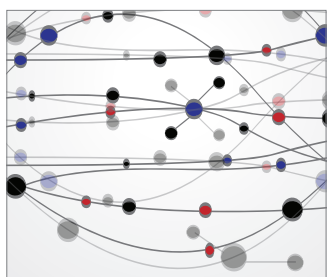

The Scientific World Journal
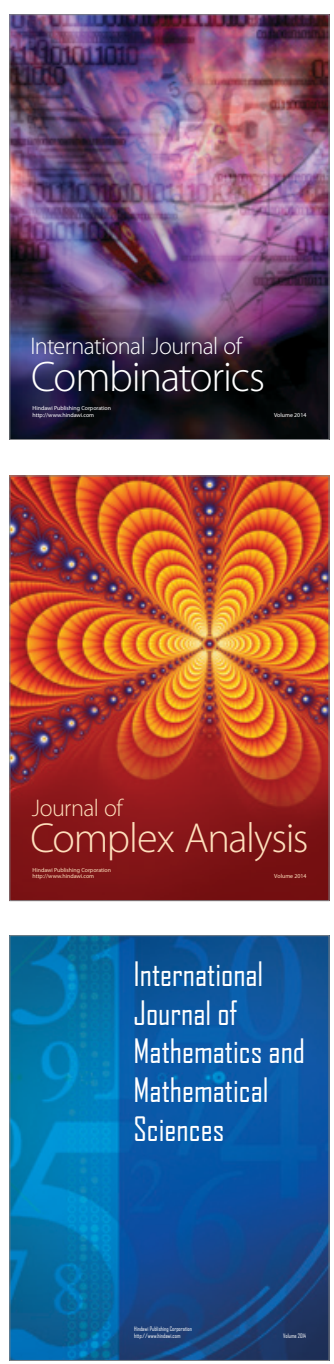
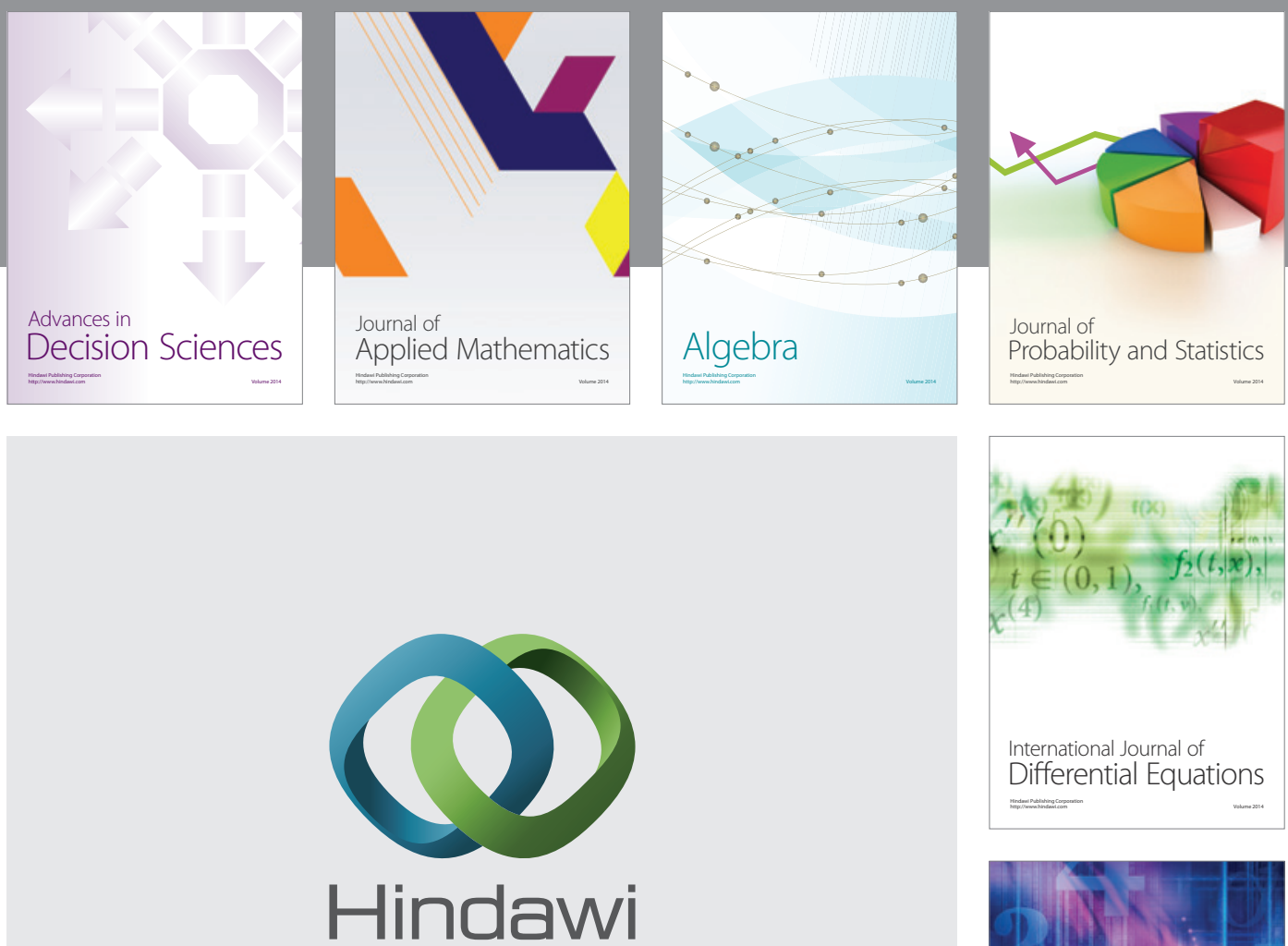

Submit your manuscripts at http://www.hindawi.com
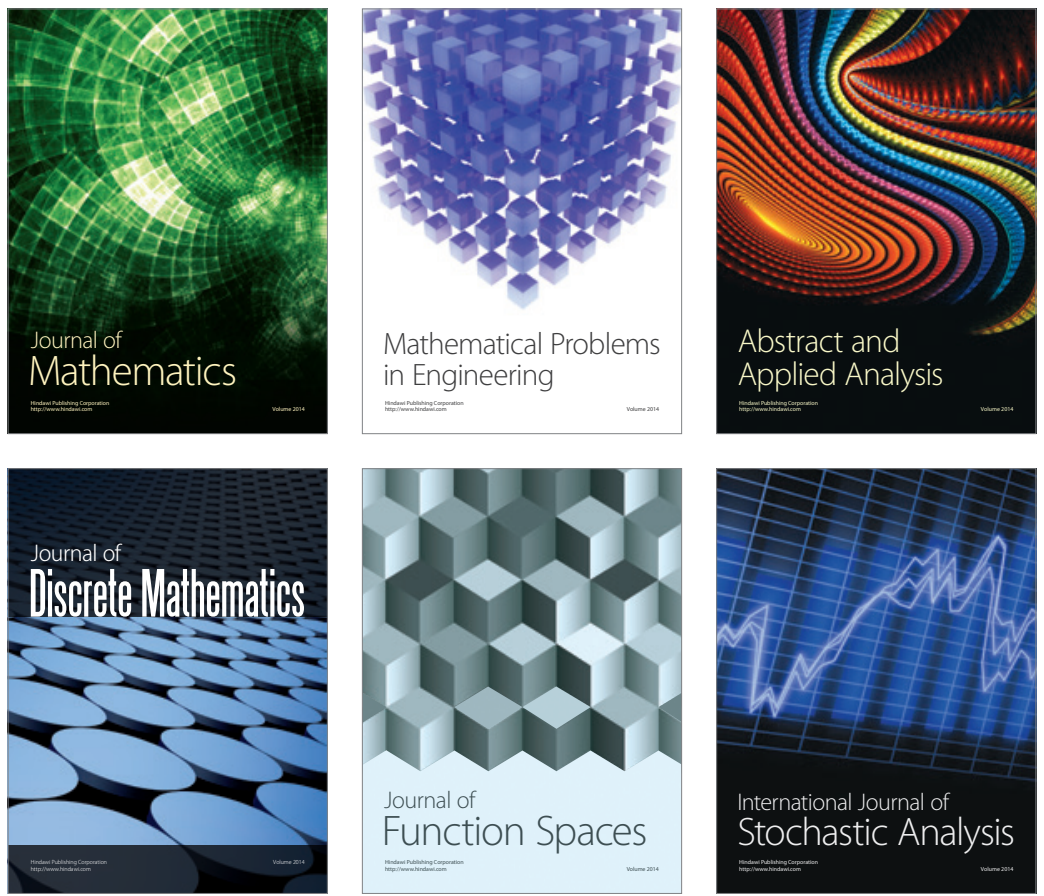

Journal of

Function Spaces

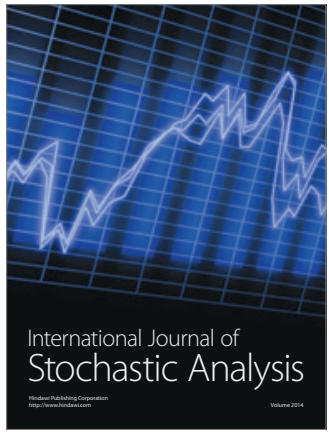

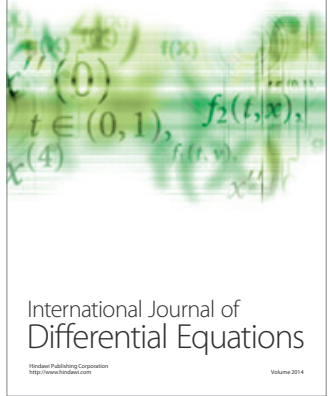
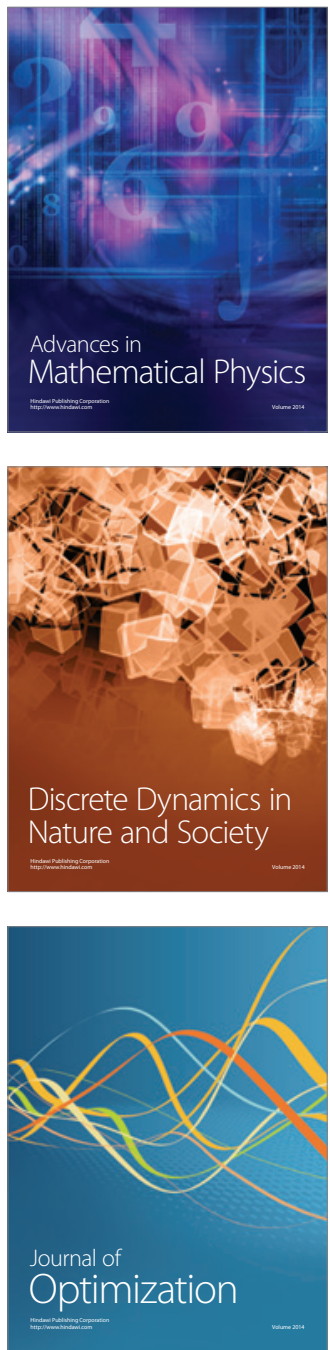